\title{
Article
}

\section{Some New Reverse Hilbert's Inequalities on Time Scales}

\author{
Ghada AlNemer ${ }^{1}(0)$, Ahmed I. Saied ${ }^{2}$, Mohammed Zakarya ${ }^{3,4}$, Hoda A. Abd El-Hamid ${ }^{5}$, Omar Bazighifan ${ }^{6,7, *(1)}$ \\ and Haytham M. Rezk ${ }^{8}$ (D)
}

1 Department of Mathematical Science, College of Science, Princess Nourah Bint Abdulrahman University, P.O. Box 105862, Riyadh 11656, Saudi Arabia; gnnemer@pnu.edu.sa

2 Department of Mathematics, Faculty of Science, Benha University, Benha 13511, Egypt; as0863289@gmail.com

3 Department of Mathematics, College of Science, King Khalid University, P.O. Box 9004,

Abha 61413, Saudi Arabia; Mohammed_Zakaria1983@yahoo.com or mzibrahim@kku.edu.sa

4 Department of Mathematics, Faculty of Science, Al-Azhar University, Assiut 71524, Egypt

5 Department of Mathematics and Computer Science, Faculty of Science, Beni-Suef University,

Beni-Suef 62511, Egypt; houdamaths4@gmail.com

6 Section of Mathematics, International Telematic University Uninettuno, Corso Vittorio Emanuele II, 39, 00186 Rome, Italy

7 Department of Mathematics, Faculty of Science, Hadhramout University, Hadhramout 50512, Yemen

8 Department of Mathematics, Faculty of Science, Al-Azhar University, Nasr City 11884, Egypt; haythamrezk@azhar.edu.eg

* Correspondence: o.bazighifan@gmail.com

check for updates

Citation: AlNemer, G.; Saied, A.I.; Zakarya, M.; Abd El-Hamid, H.A.; Bazighifan, O.; Rezk, H.M. Some New Reverse Hilbert's Inequalities on Time Scales. Symmetry 2021, 13, 2431. https://doi.org/10.3390/ sym13122431

Academic Editor: Serkan Araci

Received: 26 October 2021

Accepted: 2 December 2021

Published: 15 December 2021

Publisher's Note: MDPI stays neutral with regard to jurisdictional claims in published maps and institutional affiliations.

Copyright: (c) 2021 by the authors. Licensee MDPI, Basel, Switzerland. This article is an open access article distributed under the terms and conditions of the Creative Commons Attribution (CC BY) license (https:// creativecommons.org/licenses/by/ $4.0 /)$.
Abstract: This paper is interested in establishing some new reverse Hilbert-type inequalities, by using chain rule on time scales, reverse Jensen's, and reverse Hölder's with Specht's ratio and mean inequalities. To get the results, we used the Specht's ratio function and its applications for reverse inequalities of Hilbert-type. Symmetrical properties play an essential role in determining the correct methods to solve inequalities. The new inequalities in special cases yield some recent relevance, which also provide new estimates on inequalities of these type.

Keywords: reverse Hilbert-type inequalities; Specht's ratio; time scales; reverse Hölder inequalities

\section{Introduction}

In [1], Hardy established that

$$
\sum_{j=1}^{\infty} \sum_{i=1}^{\infty} \frac{\varphi_{i} \psi_{j}}{i+j} \leq \frac{\pi}{\sin \frac{\pi}{\alpha}}\left(\sum_{i=1}^{\infty} \varphi_{i}^{\alpha}\right)^{\frac{1}{\alpha}}\left(\sum_{j=1}^{\infty} \psi_{j}^{\beta}\right)^{\frac{1}{\beta}},
$$

where $\varphi_{i}, \psi_{j} \geq 0$ with $0<\sum_{i=1}^{\infty} \varphi_{i}^{\alpha}<\infty, 0<\sum_{j=1}^{\infty} \psi_{j}^{\beta}<\infty$ and $\alpha>1,1 / \alpha+1 / \beta=1$. Hardy and Reisz [2] established the continuous form of (1) in the following

$$
\int_{0}^{\infty} \int_{0}^{\infty} \frac{\delta(\chi) \omega(z)}{\chi+z} d \chi d z \leq \frac{\pi}{\sin \frac{\pi}{\alpha}}\left(\int_{0}^{\infty} \delta^{\alpha}(\chi) d \chi\right)^{\frac{1}{\alpha}}\left(\int_{0}^{\infty} \omega^{\beta}(z) d z\right)^{\frac{1}{\beta}},
$$

where $\delta$ and $\omega$ are measurable nonnegative functions such that $0<\int_{0}^{\infty} \delta^{\alpha}(\chi) d \chi<\infty$, $0<\int_{0}^{\infty} \omega^{\beta}(z) d z<\infty$ and $\pi / \sin (\pi / \alpha)$ in (1), and (2) is the best value. In [2], Hardy showed that, if $\alpha>1, \beta>1,1 / \alpha+1 / \beta \geq 1$ and $0<\lambda=2-(1 / \alpha+1 / \beta) \leq 1$, then

$$
\sum_{j=1}^{\infty} \sum_{i=1}^{\infty} \frac{\varphi_{i} \psi_{j}}{(i+j)^{\lambda}} \leq K\left(\sum_{i=1}^{\infty} \varphi_{i}^{\alpha}\right)^{\frac{1}{\alpha}}\left(\sum_{j=1}^{\infty} \psi_{j}^{\beta}\right)^{\frac{1}{\beta}}
$$


where $K=K(\alpha, \beta)$ relates to $\alpha, \beta$, only for $1 / \alpha+1 / \beta=1, \lambda=2-(1 / \alpha+1 / \beta)=1$ and the constant factor $K$ is optimal. For more details about the Hilbert-type inequalities, see the papers [3-6]. In [7], Hölder proved that

$$
\sum_{k=1}^{n} \zeta_{k} y_{k} \leq\left(\sum_{k=1}^{n} \zeta_{k}^{\alpha}\right)^{\frac{1}{\alpha}}\left(\sum_{k=1}^{n} y_{k}^{\beta}\right)^{\frac{1}{\beta}}
$$

where $\left(\zeta_{k}\right)$ and $\left(y_{k}\right)$ are positive sequences and $\alpha, \beta>1$ such that $1 / \alpha+1 / \beta=1$. The integral form of (4) is

$$
\int_{a}^{b} \psi(\tau) \omega(\tau) d \tau \leq\left(\int_{a}^{b} \psi^{\alpha}(\tau) d \tau\right)^{\frac{1}{\alpha}}\left(\int_{a}^{b} \omega^{\beta}(\tau) d \tau\right)^{\frac{1}{\beta}},
$$

where $\alpha, \beta>1$ such that $1 / \alpha+1 / \beta=1$ and $\psi, \omega \in C\left((a, b), \mathbb{R}^{+}\right)$.

Some authors established the reverse Hölder inequalities, the reverse Young inequalities, and the reverse Hilbert inequalities by using the Specht's ratio function, see [8-12]. In particular, Zhao and Cheung [11] established the reverse Hölder inequalities by using the Specht's ratio function and proved that if $\psi(\zeta)$ and $\omega(\zeta)$ are nonnegative continuous functions and $\psi^{1 / \alpha}(\zeta) \omega^{1 / \beta}(\zeta)$ is integrable on $[a, b]$, then

$$
\left(\int_{a}^{b} \psi^{\alpha}(\zeta) d \zeta\right)^{\frac{1}{\alpha}}\left(\int_{a}^{b} \omega^{\beta}(\zeta) d \zeta\right)^{\frac{1}{\beta}} \leq \int_{a}^{b} S\left(\frac{Y \psi^{\alpha}(\zeta)}{X \omega^{\beta}(\zeta)}\right) \cdot \psi(\zeta) \omega(\zeta) d \zeta
$$

with

$$
X=\int_{a}^{b} \psi^{\alpha}(\zeta) d \zeta, Y=\int_{a}^{b} \omega^{\beta}(\zeta) d \zeta, \alpha>1 \text { and } \frac{1}{\alpha}+\frac{1}{\beta}=1,
$$

where the function $S($.$) is called the Specht's ratio function (see [10]) and defined as follows:$

$$
S(h)=\frac{h^{1 /(h-1)}}{e \log h^{1 /(h-1)}}, h \neq 1 \text { and } S(1)=1 .
$$

In [11], the authors proved that if $\psi, \omega \in C\left((a, b), \mathbb{R}^{+}\right)$and $m>0$, then

$$
\int_{a}^{b} \frac{\psi^{m+1}(\zeta)}{\mathscr{\omega}^{m}(\zeta)} d \zeta \leq \frac{\left(\int_{a}^{b} S\left(\frac{G \psi^{m+1}(\zeta)}{F \mathscr{\omega}^{m+1}(\zeta)}\right) \psi(\zeta) d \zeta\right)^{m+1}}{\left(\int_{a}^{b} \mathscr{\omega}(\zeta) d \zeta\right)^{m}}
$$

where

$$
G=\int_{a}^{b} \omega(\zeta) d \zeta \text { and } F=\int_{a}^{b} \psi^{m+1}(\zeta) / \omega^{m}(\zeta) d \zeta
$$

In addition, they proved the discrete case of (7) as follows:

$$
\sum \frac{a_{i}^{m+1}}{b_{i}^{m}} \leq \frac{\sum S\left(\frac{B a_{i}^{m+1}}{A b_{i}^{m+1}}\right) a_{i}}{\left(\sum b_{i}\right)^{m}}
$$

where $B=\sum b_{i}$ and $A=\sum a_{i}^{m+1} / b_{i}^{m}$. 
Symmetry 2021, 13, 2431

3 of 20

In [12], Zhao and Cheung established the reverse Hilbert inequalities by using the Specht's ratio and proved that if $0 \leq \alpha, \beta \leq 1,\left\{\lambda_{i}\right\},\left\{\psi_{j}\right\}$ are nonnegative and decreasing sequences of real numbers for $i=1,2, \ldots, k$ and $j=1,2, \ldots, r$ with $k, r \in \mathbb{N}$, then

$$
\begin{aligned}
& \sum_{i=1}^{k} \sum_{j=1}^{r} \frac{S_{\alpha, \beta, k, r, i, j}\left(\sum_{s=1}^{i} \lambda_{s}\right)^{\alpha}\left(\sum_{t=1}^{j} \psi_{t}\right)^{\beta}}{(i j)^{\frac{1}{2}}} \\
\geq & 2 D(\alpha, \beta, k, r)\left(\sum_{i=1}^{k}\left[\lambda_{i}\left(\sum_{s=1}^{i} \lambda_{s}\right)^{\alpha-1}\right]^{2}(k-i+1)\right)^{\frac{1}{2}} \\
& \times\left(\sum_{j=1}^{r}\left[\psi_{j}\left(\sum_{t=1}^{j} \psi_{t}\right)^{\beta-1}\right]^{2}(r-j+1)\right)^{\frac{1}{2}}
\end{aligned}
$$

where

$$
D(\alpha, \beta, k, r)=\frac{1}{2} \alpha \beta(k r)^{\frac{1}{2}},
$$

and

$$
\begin{aligned}
S_{\alpha, \beta, k, r, i, j} & =S\left(\frac{k \sum_{s=1}^{i}\left[\lambda_{s}\left(\sum_{\tau=1}^{s} \lambda_{\tau}\right)^{\alpha-1}\right]^{2}}{\sum_{s=1}^{k}(k-s+1)\left[\lambda_{s}\left(\sum_{\tau=1}^{s} \lambda_{\tau}\right)^{\alpha-1}\right]^{2}}\right) \\
& \times S\left(\frac{r \sum_{t=1}^{j}\left[\psi_{t}\left(\sum_{\tau=1}^{t} \psi_{\tau}\right)^{\beta-1}\right]^{2}}{\sum_{t=1}^{r}(r-t+1)\left[\psi_{t}\left(\sum_{\tau=1}^{t} \psi_{\tau}\right)^{\beta-1}\right]^{2}}\right) \\
& \times S\left(\frac{i\left[\lambda_{u}\left(\sum_{\tau=1}^{u} \lambda_{\tau}\right)^{\alpha-1}\right]^{2}}{\sum_{s=1}^{i}\left[\lambda_{s}\left(\sum_{\tau=1}^{s} \lambda_{\tau}\right)^{\alpha-1}\right]^{2}}\right) S\left(\frac{j\left[\psi_{v}\left(\sum_{\tau=1}^{v} \psi_{\tau}\right)^{\beta-1}\right]^{2}}{\sum_{t=1}^{j}\left[\psi_{t}\left(\sum_{\tau=1}^{t} \psi_{\tau}\right)^{\beta-1}\right]^{2}}\right)
\end{aligned}
$$

where

$$
\begin{aligned}
& S\left(\frac{i\left[\lambda_{u}\left(\sum_{\tau=1}^{u} \lambda_{\tau}\right)^{\alpha-1}\right]^{2}}{\sum_{s=1}^{i}\left[\lambda_{s}\left(\sum_{\tau=1}^{s} \lambda_{\tau}\right)^{\alpha-1}\right]^{2}}\right) \\
= & \max \left\{S\left(\frac{i\left[\lambda_{1}\left(\sum_{\tau=1}^{1} \lambda_{\tau}\right)^{\alpha-1}\right]^{2}}{\sum_{s=1}^{i}\left[\lambda_{s}\left(\sum_{\tau=1}^{s} \lambda_{\tau}\right)^{\alpha-1}\right]^{2}}\right) ; S\left(\frac{i\left[\lambda_{i}\left(\sum_{\tau=1}^{i} \lambda_{\tau}\right)^{\alpha-1}\right]^{2}}{\sum_{s=1}^{i}\left[\lambda_{s}\left(\sum_{\tau=1}^{s} \lambda_{\tau}\right)^{\alpha-1}\right]^{2}}\right)\right\},
\end{aligned}
$$

and

$$
\begin{aligned}
& S\left(\frac{j\left[\psi_{v}\left(\sum_{\tau=1}^{v} \psi_{\tau}\right)^{\beta-1}\right]^{2}}{\sum_{t=1}^{j}\left[\psi_{t}\left(\sum_{\tau=1}^{t} \psi_{\tau}\right)^{\beta-1}\right]^{2}}\right) \\
= & \max \left\{S\left(\frac{j\left[\psi_{1}\left(\sum_{\tau=1}^{1} \psi_{\tau}\right)^{\beta-1}\right]^{2}}{\sum_{t=1}^{j}\left[\psi_{t}\left(\sum_{\tau=1}^{t} \psi_{\tau}\right)^{\beta-1}\right]^{2}}\right) ; S\left(\frac{j\left[\psi_{j}\left(\sum_{\tau=1}^{j} \psi_{\tau}\right)^{\beta-1}\right]^{2}}{\sum_{t=1}^{j}\left[\psi_{t}\left(\sum_{\tau=1}^{t} \psi_{\tau}\right)^{\beta-1}\right]^{2}}\right)\right\},
\end{aligned}
$$


In addition, they proved that, if $\left\{\lambda_{i}\right\},\left\{\omega_{j}\right\}$ are nonnegative sequences for $i=1,2, \ldots, k$, and $j=1,2, \ldots, r$ with $k, r \in \mathbb{N}$ and $\left\{\alpha_{i}\right\},\left\{\beta_{j}\right\}$ are positive sequences. Let $\phi, \psi$ are nonnegative, concave and supermultiplicative functions. Then,

$$
\begin{aligned}
& \sum_{i=1}^{k} \sum_{j=1}^{r} \frac{S_{k, r, i, j} \phi\left(\Lambda_{i}\right) \psi\left(\Omega_{j}\right)}{(i j)^{\frac{1}{2}}} \\
\geq & 2 M(k, r)\left(\sum_{s=1}^{k}\left[\alpha_{s} \phi\left(\frac{\lambda_{s}}{\alpha_{s}}\right)\right]^{2}(k-s+1)\right)^{\frac{1}{2}} \\
& \times\left(\sum_{t=1}^{r}\left[\beta_{t} \psi\left(\frac{\omega_{t}}{\beta_{t}}\right)\right]^{2}(r-t+1)\right)^{\frac{1}{2}}
\end{aligned}
$$

with

$$
\begin{gathered}
M(k, r)=\frac{1}{2}\left(\sum_{i=1}^{k}\left(\frac{\phi\left(\alpha_{i}\right)}{\alpha_{i}}\right)^{2}\right)^{\frac{1}{2}}\left(\sum_{j=1}^{r}\left(\frac{\psi\left(Q_{j}\right)}{Q_{j}}\right)^{2}\right)^{\frac{1}{2}}, \\
S_{k, r, i, j}=S\left(\frac{\left(\sum_{s=1}^{k}\left[\alpha_{s} \phi\left(\frac{\lambda_{s}}{\alpha_{s}}\right)\right]^{2}(k-s+1)\right)\left(\frac{\phi\left(\alpha_{j}\right)}{\alpha_{i}}\right)^{2}}{\left(\sum_{i=1}^{k}\left(\frac{\phi\left(\alpha_{i}\right)}{\alpha_{i}}\right)^{2}\right)\left(\sum_{s=1}^{i}\left[\alpha_{s} \phi\left(\frac{\lambda_{s}}{\alpha_{s}}\right)\right]^{2}\right)}\right) S\left(\frac{\left(\sum_{t=1}^{r}\left[\beta_{t} \psi\left(\frac{\omega_{t}}{\beta_{t}}\right)\right]^{2}(r-t+1)\right)\left(\frac{\psi\left(Q_{j}\right)}{Q_{j}}\right)^{2}}{\left(\sum_{j=1}^{r}\left(\frac{\psi\left(Q_{j}\right)}{Q_{j}}\right)^{2}\right)\left(\sum_{t=1}^{j}\left[\beta_{t} \psi\left(\frac{\omega_{t}}{\beta_{t}}\right)\right]^{2}\right)}\right), \\
\Lambda_{i}=\sum_{s=1}^{i} S\left(\frac{i\left[\alpha_{s} \phi\left(\frac{\lambda_{s}}{\alpha_{s}}\right)\right]^{2}}{\sum_{s=1}^{i}\left[\alpha_{s} \phi\left(\frac{\lambda_{s}}{\alpha_{s}}\right)\right]^{2}}\right) \lambda_{s}, \Omega_{j}=\sum_{t=1}^{j} S\left(\frac{j\left[\beta_{t} \psi\left(\frac{\omega_{t}}{\beta_{t}}\right)\right]^{2}}{\sum_{t=1}^{j}\left[\beta_{t} \psi\left(\frac{\omega_{t}}{\beta_{t}}\right)\right]^{2}}\right) \omega_{t},
\end{gathered}
$$

and

$$
\alpha_{i}=\sum_{s=1}^{i} S\left(\frac{i\left[\alpha_{s} \phi\left(\frac{\lambda_{s}}{\alpha_{s}}\right)\right]^{2}}{\sum_{s=1}^{i}\left[\alpha_{s} \phi\left(\frac{\lambda_{s}}{\alpha_{s}}\right)\right]^{2}}\right) \alpha_{s}, Q_{j}=\sum_{t=1}^{j} S\left(\frac{j\left[\beta_{t} \psi\left(\frac{\omega_{t}}{\beta_{t}}\right)\right]^{2}}{\sum_{t=1}^{j}\left[\beta_{t} \psi\left(\frac{\omega_{t}}{\beta_{t}}\right)\right]^{2}}\right) \beta_{t},
$$

where the function $S($.$) is the Specht's ratio. In [12], the authors proved that, if \left\{\lambda_{i}\right\},\left\{\omega_{j}\right\}$ are nonnegative sequences for $i=1,2, \ldots, k$ and $j=1,2, \ldots, r$ with $k, r \in \mathbb{N}$, then

$$
\begin{aligned}
& \sum_{i=1}^{k} \sum_{j=1}^{r} \frac{S_{k, r, i, j} \Lambda_{i} \Omega_{j}}{(i j)^{\frac{1}{2}}} \\
\geq & (k r)^{\frac{1}{2}}\left(\sum_{i=1}^{k} \lambda_{i}^{2}(k-i+1)\right)^{\frac{1}{2}}\left(\sum_{j=1}^{r} \omega_{j}^{2}(r-j+1)\right)^{\frac{1}{2}},
\end{aligned}
$$

with

$$
\begin{aligned}
& S_{k, r, i, j}=S\left(\frac{\sum_{s=1}^{k} \lambda_{s}^{2}(k-s+1)}{k\left(\sum_{s=1}^{i} \lambda_{s}^{2}\right)}\right) S\left(\frac{\sum_{t=1}^{r} \omega_{t}^{2}(r-t+1)}{r\left(\sum_{t=1}^{j} \omega_{t}^{2}\right)}\right), \\
& \Lambda_{i}=\sum_{s=1}^{i} S\left(\frac{i \lambda_{s}^{2}}{\sum_{s=1}^{i} \lambda_{s}^{2}}\right) \lambda_{s} \text { and } \Omega_{j}=\sum_{t=1}^{j} S\left(\frac{j \omega_{t}^{2}}{\sum_{t=1}^{j} \omega_{t}^{2}}\right) \omega_{t} .
\end{aligned}
$$

In the last few decades, a new theory has been discovered to unify the continuous calculus and discrete calculus. It is called a time scale theory. A time scale $\mathbb{T}$ is an arbitrary nonempty closed subset of the real numbers $\mathbb{R}$. Many authors established dynamic inequalities and generalized them on time scales. For more details, see ([13-17]).

In particular, El-Deeb, Elsennary, and Wing-Sum Cheung [15] proved the reverse Hölder inequality on time scales by using the Specht's ratio function and proved that, 
if $\psi, \omega \in C\left([a, b]_{\mathbb{T}}, \mathbb{R}^{+}\right)$such that $\psi^{\alpha}, \omega^{\beta}$ are $\diamond_{\alpha}-$ integrable on $[a, b]_{\mathbb{T}}$. If $\alpha>1$ and $1 / \alpha+1 / \beta=1$, then

$$
\begin{gathered}
\int_{a}^{b} S\left(\frac{Y \psi^{\alpha}(\zeta)}{X \omega^{\beta}(\zeta)}\right) \psi(\zeta) \omega(\zeta) \diamond_{\alpha} \zeta \\
\geq\left(\int_{a}^{b} \psi^{\alpha}(\zeta) \diamond_{\alpha} \zeta\right)^{\frac{1}{\alpha}}\left(\int_{a}^{b} \omega^{\beta}(\zeta) \diamond_{\alpha} \zeta\right)^{\frac{1}{\beta}},
\end{gathered}
$$

where $X=\int_{a}^{b} \psi^{\alpha}(\zeta) \nabla_{\alpha} \zeta, Y=\int_{a}^{b} \omega^{\beta}(\zeta) \diamond_{\alpha} \zeta$ and $S($.$) is the Specht's ratio (see [11]). In addi-$ tion, they proved (12) with weighted functions and proved that if $\psi, \omega, w \in C\left([a, b]_{\mathbb{T}}, \mathbb{R}^{+}\right)$ such that $\psi^{\alpha}, \omega^{\beta}$ are $\nabla_{\alpha}-$ integrable on $[a, b]_{\mathbb{T}}$. If $\alpha>1$ and $1 / \alpha+1 / \beta=1$, then

$$
\begin{aligned}
& \int_{a}^{b} S\left(\frac{Y \psi^{\alpha}(\zeta)}{X \omega^{\beta}(\zeta)}\right) \cdot w(\zeta) \psi(\zeta) \omega(\zeta) \diamond_{\alpha} \zeta \\
\geq & \left(\int_{a}^{b} w(\zeta) \psi^{\alpha}(\zeta) \diamond_{\alpha} \zeta\right)^{\frac{1}{\alpha}}\left(\int_{a}^{b} w(\zeta) \omega^{\beta}(\zeta) \diamond_{\alpha} \zeta\right)^{\frac{1}{\beta}},
\end{aligned}
$$

where $X=\int_{a}^{b} w(\zeta) \psi^{\alpha}(\zeta) \diamond_{\alpha} \zeta$ and $Y=\int_{a}^{b} w(\zeta) \omega^{\beta}(\zeta) \diamond_{\alpha}$.

The authors [15] proved that if $\psi, \omega \in C\left([a, b]_{\mathbb{T}}, \mathbb{R}^{+}\right)$such that $0<m \leq \psi(t) / \omega(t) \leq$ $M<\infty$ for all $t \in[a, b]_{\mathbb{T}}$. If $\alpha>1$ and $1 / \alpha+1 / \beta=1$, then

$$
\begin{aligned}
& \int_{a}^{b} S\left(\frac{Y \psi(\zeta)}{X \omega(\zeta)}\right) \psi^{\frac{1}{\alpha}}(\zeta) \omega^{\frac{1}{\beta}}(\zeta) \nabla_{\alpha} \zeta \\
\geq & \frac{m^{\frac{1}{\alpha^{2}}}}{M^{\frac{1}{\beta^{2}}}} \int_{a}^{b} \psi^{\frac{1}{\beta}}(\zeta) \omega^{\frac{1}{\alpha}}(\zeta) \diamond_{\alpha} \zeta,
\end{aligned}
$$

where $X=\int_{a}^{b} \psi(\zeta) \diamond_{\alpha} \zeta$ and $Y=\int_{a}^{b} \omega(\zeta) \diamond_{\alpha} \zeta$.

The aim of this paper is to establish some new reverse Hilbert-type inequalities on time scales by using the Specht's ratio function and applying reverse Hölder inequalities on time scales.

The organization of the paper is as follows: in Section 2, we show some basics of the time scale theory and some lemmas on time scales needed in Section 3 where we prove our results. Our main results (when $\mathbb{T}=\mathbb{R}$ ) give the inequalities (9)-(11) proved by Zhao and Cheung [12].

\section{Preliminaries and Basic Lemmas}

A forward jump operator on time scales is defined by: $\sigma(\tau):=\inf \{r \in \mathbb{T}: r>\tau\}$. The set of all such rd-continuous functions is ushered by $C_{r d}(\mathbb{T}, \mathbb{R})$ and for any function $\Phi: \mathbb{T} \rightarrow \mathbb{R}$ the notation $\Phi^{\sigma}(\tau)$ denotes $\Phi(\sigma(\tau))$. To learn more about the time scale calculus, see $([18,19])$.

The derivative of the product $\Phi \omega$ and the quotient $\Phi / \omega\left(\right.$ where $\omega \omega^{\sigma} \neq 0$ ) of two differentiable functions $\Phi$ and $\omega$ are given by

$$
(\Phi \omega)^{\Delta}=\Phi^{\Delta} \omega+\Phi^{\sigma} \mathcal{\omega}^{\Delta}=\Phi \mathcal{\omega}^{\Delta}+\Phi^{\Delta} \mathcal{W}^{\sigma},\left(\frac{\Phi}{\omega}\right)^{\Delta}=\frac{\Phi^{\Delta} \mathscr{\omega}-\Phi \mathcal{\omega}^{\Delta}}{\mathcal{\omega} \mathcal{\omega}^{\sigma}} .
$$

The integration by parts formula on time scales is given by

$$
\int_{v_{0}}^{v} \lambda(\tau) \varphi^{\Delta}(\tau) \Delta \tau=[\lambda(\tau) \varphi(\tau)]_{v_{0}}^{v}-\int_{v_{0}}^{v} \lambda^{\Delta}(\tau) \varphi^{\sigma}(\tau) \Delta \tau
$$

The time scales chain rule (see Theorem 1.87 [18]) is as follows:

$$
(\omega \circ \varphi)^{\Delta}(\tau)=\omega^{\prime}(\varphi(\chi)) \varphi^{\Delta}(\tau), \text { where } \chi \in[\tau, \sigma(\tau)],
$$


and $\omega: \mathbb{R} \rightarrow \mathbb{R}$ is continuously differentiable, $\varphi: \mathbb{T} \rightarrow \mathbb{R}$ is $\Delta$ - differentiable.

Definition 1 ([20]). A function $h: J \subset \mathbb{R} \rightarrow \mathbb{R}^{+}$is supermultiplicative if

$$
h(\chi z) \geq h(\chi) h(z), \quad \forall \chi, z \in J .
$$

The inequality (18) holds with equality when $h$ is the identity map (i.e., $h(\chi)=\chi$ ). If the last inequality has a reversed sign, then $h$ is said to be a submultiplicative function.

Lemma 1. Let $\mathbb{T}$ be a time scale with $a \in \mathbb{T}, \lambda$ is nonnegative $r d$-continuous function and $0<\gamma \leq 1$, then

$$
\left(\int_{a}^{\sigma(t)} \lambda(\tau) \Delta \tau\right)^{\gamma} \geq \gamma \int_{a}^{\sigma(t)}\left(\int_{a}^{\sigma(\chi)} \lambda(\tau) \Delta \tau\right)^{\gamma-1} \lambda(\chi) \Delta \chi
$$

Proof. By applying (17) on the term $\int_{a}^{\chi} \lambda(\tau) \Delta \tau$, we get

$$
\left[\left(\int_{a}^{\chi} \lambda(\tau) \Delta \tau\right)^{\gamma}\right]^{\Delta}=\gamma\left(\int_{a}^{\zeta} \lambda(\tau) \Delta \tau\right)^{\gamma-1} \lambda(\chi), \quad \zeta \in[\chi, \sigma(\chi)] .
$$

Since $\zeta \leq \sigma(\chi)$, then we have (note $0<\gamma \leq 1$ ) that

$$
\left(\int_{a}^{\zeta} \lambda(\tau) \Delta \tau\right)^{\gamma-1} \geq\left(\int_{a}^{\sigma(\chi)} \lambda(\tau) \Delta \tau\right)^{\gamma-1}
$$

Substituting (21) into (20), we see that

$$
\left[\left(\int_{a}^{\chi} \lambda(\tau) \Delta \tau\right)^{\gamma}\right]^{\Delta} \geq \gamma\left(\int_{a}^{\sigma(\chi)} \lambda(\tau) \Delta \tau\right)^{\gamma-1} \lambda(\chi)
$$

Integrating the last inequality over $\chi$ from $a$ to $\sigma(t)$, we observe that

$$
\int_{a}^{\sigma(t)}\left[\left(\int_{a}^{\chi} \lambda(\tau) \Delta \tau\right)^{\gamma}\right]^{\Delta} \Delta \chi \geq \gamma \int_{a}^{\sigma(t)}\left(\int_{a}^{\sigma(\chi)} \lambda(\tau) \Delta \tau\right)^{\gamma-1} \lambda(\chi) \Delta \chi .
$$

i.e.,

$$
\left(\int_{a}^{\sigma(t)} \lambda(\tau) \Delta \tau\right)^{\gamma} \geq \gamma \int_{a}^{\sigma(t)}\left(\int_{a}^{\sigma(\chi)} \lambda(\tau) \Delta \tau\right)^{\gamma-1} \lambda(\chi) \Delta \chi
$$

which is (19). The proof is complete.

Lemma 2 (Specht's ratio [10]). If $\alpha$ and $\beta$ are positive numbers, $p>1$ and $1 / p+1 / q=1$, then

$$
S\left(\frac{\alpha}{\beta}\right) \alpha^{1 / p} \beta^{1 / q} \geq \frac{\alpha}{p}+\frac{\beta}{q}
$$

where

$$
S(h)=\frac{h^{1 /(h-1)}}{e \log h^{1 /(h-1)}}, h \neq 1 .
$$

Lemma 3 ([10]). Let $S($.$) be a Specht's ratio function which is defined in Lemma 2, then the$ function $S(t)$ is strictly decreasing for $0<t<1$ and strictly increasing for $t>1$. Furthermore, the following equations hold:

$$
S(1)=1 \text { and } S(t)=S\left(\frac{1}{t}\right) \text { for all } t>0 .
$$

In [15] for $\alpha=1$, we get the following lemma. 
Lemma 4. If $\delta, \omega \in C\left([a, b]_{\mathbb{T}}, \mathbb{R}^{+}\right)$such that $\delta^{\gamma}$, $\omega^{v}$ are $\Delta$-integrable on $[a, b]_{\mathbb{T}}$. If $\gamma>1$ and $1 / \gamma+1 / v=1$, then

$$
\begin{gathered}
\int_{a}^{b} S\left(\frac{Y \delta^{\gamma}(\zeta)}{X \omega^{\nu}(\zeta)}\right) \delta(\zeta) \omega(\zeta) \Delta \zeta \\
\geq\left(\int_{a}^{b} \delta^{\gamma}(\zeta) \Delta \zeta\right)^{\frac{1}{\gamma}}\left(\int_{a}^{b} \omega^{\nu}(\zeta) \Delta \zeta\right)^{\frac{1}{v}},
\end{gathered}
$$

where $X=\int_{a}^{b} \delta^{\gamma}(\zeta) \Delta \zeta, Y=\int_{a}^{b} \omega^{\nu}(\zeta) \Delta \zeta$ and $S($.$) is the Specht's ratio.$

Theorem 1 (Jensen's inequality). Assume that $\mathbb{T}$ is a time scale with $\zeta_{0}, \zeta \in \mathbb{T}$ and $r_{0}, r \in \mathbb{R}$. If $\lambda \in \mathrm{C}_{r d}\left(\left[\zeta_{0}, \zeta\right]_{\mathbb{T}}, \mathbb{R}\right), \varphi:\left[\zeta_{0}, \zeta\right]_{\mathbb{T}} \rightarrow\left(r_{0}, r\right)$ is $r d$-continuous and $\Psi:\left(r_{0}, r\right) \rightarrow \mathbb{R}$ is continuous and convex, then

$$
\Psi\left(\frac{1}{\int_{\zeta_{0}}^{\zeta} \lambda(\tau) \Delta \tau} \int_{\zeta_{0}}^{\zeta} \lambda(\tau) \varphi(\tau) \Delta \tau\right) \leq \frac{1}{\int_{\zeta_{0}}^{\zeta} \lambda(\tau) \Delta \tau} \int_{\zeta_{0}}^{\zeta} \lambda(\tau) \Psi(\varphi(\tau)) \Delta \tau .
$$

Lemma 5. Let $a \in \mathbb{T}, \lambda, \psi$ be nonnegative and decreasing functions and $0<\alpha, \beta \leq 1$. Then,

$$
\begin{aligned}
& S\left(\frac{(\sigma(t)-a)\left[\lambda(\zeta)\left(\int_{a}^{\sigma(\zeta)} \lambda(\tau) \Delta \tau\right)^{\alpha-1}\right]^{2}}{\int_{a}^{\sigma(t)}\left[\lambda(\chi)\left(\int_{a}^{\sigma(x)} \lambda(\tau) \Delta \tau\right)^{\alpha-1}\right]^{2} \Delta \chi}\right) \\
= & \max \left\{S\left(\frac{(\sigma(t)-a)\left[\lambda(a)\left(\int_{a}^{\sigma(a)} \lambda(\tau) \Delta \tau\right)^{\alpha-1}\right]^{2}}{\int_{a}^{\sigma(t)}\left[\lambda(\chi)\left(\int_{a}^{\sigma(x)} \lambda(\tau) \Delta \tau\right)^{\alpha-1}\right]^{2} \Delta \chi}\right)\right. \\
& \left.; S\left(\frac{(\sigma(t)-a)\left[\lambda(t)\left(\int_{a}^{\sigma(t)} \lambda(\tau) \Delta \tau\right)^{\alpha-1}\right]^{2}}{\int_{a}^{\sigma(t)}\left[\lambda(\chi)\left(\int_{a}^{\sigma(x)} \lambda(\tau) \Delta \tau\right)^{\alpha-1}\right]^{2} \Delta \chi}\right)\right\}
\end{aligned}
$$

and

$$
\begin{aligned}
& S\left(\frac{(\sigma(\tilde{\xi})-a)\left[\psi(\eta)\left(\int_{a}^{\sigma(\eta)} \psi(\tau) \Delta \tau\right)^{\beta-1}\right]^{2}}{\int_{a}^{\sigma(\tilde{\xi})}\left[\psi(z)\left(\int_{a}^{\sigma(z)} \psi(\tau) \Delta \tau\right)^{\beta-1}\right]^{2} \Delta z}\right) \\
= & \max \left\{S\left(\frac{(\sigma(\xi)-a)\left[\psi(a)\left(\int_{a}^{\sigma(a)} \psi(\tau) \Delta \tau\right)^{\beta-1}\right]^{2}}{\int_{a}^{\sigma(\xi)}\left[\psi(z)\left(\int_{a}^{\sigma(z)} \psi(\tau) \Delta \tau\right)^{\beta-1}\right]^{2} \Delta z}\right)\right. \\
& \left.; S\left(\frac{(\sigma(\xi)-a)\left[\psi(\xi)\left(\int_{a}^{\sigma(\xi)} \psi(\tau) \Delta \tau\right)^{\beta-1}\right]^{2}}{\int_{a}^{\sigma(\tilde{\xi})}\left[\psi(z)\left(\int_{a}^{\sigma(z)} \psi(\tau) \Delta \tau\right)^{\beta-1}\right]^{2} \Delta z}\right)\right\}
\end{aligned}
$$

Proof. We have for $\chi \leq z$, that

$$
\int_{a}^{\sigma(\chi)} \lambda(\tau) \Delta \tau \leq \int_{a}^{\sigma(z)} \lambda(\tau) \Delta \tau
$$

and then (where $0<\alpha \leq 1$ )

$$
\left(\int_{a}^{\sigma(\chi)} \lambda(\tau) \Delta \tau\right)^{\alpha-1} \geq\left(\int_{a}^{\sigma(z)} \lambda(\tau) \Delta \tau\right)^{\alpha-1}
$$


Since $\lambda$ is decreasing, we have that

$$
\left[\lambda(\chi)\left(\int_{a}^{\sigma(\chi)} \lambda(\tau) \Delta \tau\right)^{\alpha-1}\right]^{2} \geq\left[\lambda(z)\left(\int_{a}^{\sigma(z)} \lambda(\tau) \Delta \tau\right)^{\alpha-1}\right]^{2}
$$

thus the function $\left[\lambda(\chi)\left(\int_{a}^{\sigma(\chi)} \lambda(\tau) \Delta \tau\right)^{\alpha-1}\right]^{2}$ is decreasing. Therefore, we have for $a \leq \chi$ that

$$
\left[\lambda(a)\left(\int_{a}^{\sigma(a)} \lambda(\tau) \Delta \tau\right)^{\alpha-1}\right]^{2} \geq\left[\lambda(\chi)\left(\int_{a}^{\sigma(\chi)} \lambda(\tau) \Delta \tau\right)^{\alpha-1}\right]^{2}
$$

Integrate the last inequality over $\chi$ from $a$ to $\sigma(t)$, to get

$$
(\sigma(t)-a)\left[\lambda(a)\left(\int_{a}^{\sigma(a)} \lambda(\tau) \Delta \tau\right)^{\alpha-1}\right]^{2} \geq \int_{a}^{\sigma(t)}\left[\lambda(\chi)\left(\int_{a}^{\sigma(\chi)} \lambda(\tau) \Delta \tau\right)^{\alpha-1}\right]^{2} \Delta \chi
$$

and then

$$
\frac{(\sigma(t)-a)\left[\lambda(a)\left(\int_{a}^{\sigma(a)} \lambda(\tau) \Delta \tau\right)^{\alpha-1}\right]^{2}}{\int_{a}^{\sigma(t)}\left[\lambda(\chi)\left(\int_{a}^{\sigma(x)} \lambda(\tau) \Delta \tau\right)^{\alpha-1}\right]^{2} \Delta \chi} \geq 1
$$

Since the function $\left[\lambda(\chi)\left(\int_{a}^{\sigma(\chi)} \lambda(\tau) \Delta \tau\right)^{\alpha-1}\right]^{2}$ is decreasing, we have for $\chi \leq t$ that

$$
\left[\lambda(\chi)\left(\int_{a}^{\sigma(\chi)} \lambda(\tau) \Delta \tau\right)^{\alpha-1}\right]^{2} \geq\left[\lambda(t)\left(\int_{a}^{\sigma(t)} \lambda(\tau) \Delta \tau\right)^{\alpha-1}\right]^{2}
$$

and by integrating the last inequality over $\chi$ from $a$ to $\sigma(t)$, we get that

$$
\begin{aligned}
& \int_{a}^{\sigma(t)}\left[\lambda(\chi)\left(\int_{a}^{\sigma(\chi)} \lambda(\tau) \Delta \tau\right)^{\alpha-1}\right]^{2} \Delta \chi \\
\geq & \int_{a}^{\sigma(t)}\left[\lambda(t)\left(\int_{a}^{\sigma(t)} \lambda(\tau) \Delta \tau\right)^{\alpha-1}\right]^{2} \Delta \chi \\
= & (\sigma(t)-a)\left[\lambda(t)\left(\int_{a}^{\sigma(t)} \lambda(\tau) \Delta \tau\right)^{\alpha-1}\right]^{2},
\end{aligned}
$$

and then

$$
\frac{(\sigma(t)-a)\left[\lambda(t)\left(\int_{a}^{\sigma(t)} \lambda(\tau) \Delta \tau\right)^{\alpha-1}\right]^{2}}{\int_{a}^{\sigma(t)}\left[\lambda(\chi)\left(\int_{a}^{\sigma(\chi)} \lambda(\tau) \Delta \tau\right)^{\alpha-1}\right]^{2} \Delta \chi} \leq 1
$$

From (27) and (28), we observe that

$$
\frac{(\sigma(t)-a)\left[\lambda(a)\left(\int_{a}^{\sigma(a)} \lambda(\tau) \Delta \tau\right)^{\alpha-1}\right]^{2}}{\int_{a}^{\sigma(t)}\left[\lambda(\chi)\left(\int_{a}^{\sigma(\chi)} \lambda(\tau) \Delta \tau\right)^{\alpha-1}\right]^{2} \Delta \chi} \geq \ldots \geq 1 \geq \ldots \geq \frac{(\sigma(t)-a)\left[\lambda(t)\left(\int_{a}^{\sigma(t)} \lambda(\tau) \Delta \tau\right)^{\alpha-1}\right]^{2}}{\int_{a}^{\sigma(t)}\left[\lambda(\chi)\left(\int_{a}^{\sigma(\chi)} \lambda(\tau) \Delta \tau\right)^{\alpha-1}\right]^{2} \Delta \chi} .
$$


Since the function (Specht's ratio $S()$.$) is decreasing on (0,1)$ and increasing on $(1, \infty)$, we observe that one of

$$
S\left(\frac{(\sigma(t)-a)\left[\lambda(a)\left(\int_{a}^{\sigma(a)} \lambda(\tau) \Delta \tau\right)^{\alpha-1}\right]^{2}}{\int_{a}^{\sigma(t)}\left[\lambda(\chi)\left(\int_{a}^{\sigma(\chi)} \lambda(\tau) \Delta \tau\right)^{\alpha-1}\right]^{2} \Delta \chi}\right) \text { and } S\left(\frac{(\sigma(t)-a)\left[\lambda(t)\left(\int_{a}^{\sigma(t)} \lambda(\tau) \Delta \tau\right)^{\alpha-1}\right]^{2}}{\int_{a}^{\sigma(t)}\left[\lambda(\chi)\left(\int_{a}^{\sigma(x)} \lambda(\tau) \Delta \tau\right)^{\alpha-1}\right]^{2} \Delta \chi}\right)
$$

is maximum (where $S(1)=1$ ), and it is in the form

$$
\begin{aligned}
& S\left(\frac{(\sigma(t)-a)\left[\lambda(\zeta)\left(\int_{a}^{\sigma(\zeta)} \lambda(\tau) \Delta \tau\right)^{\alpha-1}\right]^{2}}{\int_{a}^{\sigma(t)}\left[\lambda(\chi)\left(\int_{a}^{\sigma(x)} \lambda(\tau) \Delta \tau\right)^{\alpha-1}\right]^{2} \Delta \chi}\right) \\
= & \max \left\{S\left(\frac{(\sigma(t)-a)\left[\lambda(a)\left(\int_{a}^{\sigma(a)} \lambda(\tau) \Delta \tau\right)^{\alpha-1}\right]^{2}}{\int_{a}^{\sigma(t)}\left[\lambda(\chi)\left(\int_{a}^{\sigma(x)} \lambda(\tau) \Delta \tau\right)^{\alpha-1}\right]^{2} \Delta \chi}\right)\right. \\
& \left.; S\left(\frac{(\sigma(t)-a)\left[\lambda(t)\left(\int_{a}^{\sigma(t)} \lambda(\tau) \Delta \tau\right)^{\alpha-1}\right]^{2}}{\int_{a}^{\sigma(t)}\left[\lambda(\chi)\left(\int_{a}^{\sigma(x)} \lambda(\tau) \Delta \tau\right)^{\alpha-1}\right]^{2} \Delta \chi}\right)\right\}
\end{aligned}
$$

which is (25). Similarly, with respect to the decreasing function $\psi$ when $0<\beta \leq 1$, we have

$$
\begin{aligned}
& S\left(\frac{(\sigma(\xi)-a)\left[\psi(\eta)\left(\int_{a}^{\sigma(\eta)} \psi(\tau) \Delta \tau\right)^{\beta-1}\right]^{2}}{\int_{a}^{\sigma(\xi)}\left[\psi(z)\left(\int_{a}^{\sigma(z)} \psi(\tau) \Delta \tau\right)^{\beta-1}\right]^{2} \Delta z}\right) \\
= & \max \left\{S\left(\frac{(\sigma(\xi)-a)\left[\psi(a)\left(\int_{a}^{\sigma(a)} \psi(\tau) \Delta \tau\right)^{\beta-1}\right]^{2}}{\int_{a}^{\sigma(\xi)}\left[\psi(z)\left(\int_{a}^{\sigma(z)} \psi(\tau) \Delta \tau\right)^{\beta-1}\right]^{2} \Delta z}\right)\right. \\
& \left.; S\left(\frac{(\sigma(\xi)-a)\left[\psi(\xi)\left(\int_{a}^{\sigma(\xi)} \psi(\tau) \Delta \tau\right)^{\beta-1}\right]^{2}}{\int_{a}^{\sigma(\xi)}\left[\psi(z)\left(\int_{a}^{\sigma(z)} \psi(\tau) \Delta \tau\right)^{\beta-1}\right]^{2} \Delta z}\right)\right\}
\end{aligned}
$$

which is (26).

\section{Main Results}

Throughout the paper, we will assume that the functions are nonnegative rd-continuous functions on $[a, b]_{\mathbb{T}}$ and the integrals considered are assumed to exist. We define the time scale interval $[a, b]_{\mathbb{T}}$ by $[a, b]_{\mathbb{T}}:=[a, b] \cap \mathbb{T}$.

Now, we can present and prove the first result of this section.

Theorem 2. Let $a \in \mathbb{T}, 0 \leq \alpha, \beta \leq 1, \lambda, \psi$ be nonnegative and decreasing functions. Then, the inequality

$$
\begin{aligned}
& \int_{a}^{\sigma(s)} \int_{a}^{\sigma(r)} \frac{S_{\alpha, \beta, t, \xi, r, s}\left(\int_{a}^{\sigma(t)} \lambda(\tau) \Delta \tau\right)^{\alpha}\left(\int_{a}^{\sigma(\xi)} \psi(\tau) \Delta \tau\right)^{\beta}}{(\sigma(t)-a)^{\frac{1}{2}}(\sigma(\xi)-a)^{\frac{1}{2}}} \Delta t \Delta \xi \\
\geq & 2 C(\alpha, \beta, r, s)\left(\int_{a}^{\sigma(r)}\left[\lambda(t)\left(\int_{a}^{\sigma(t)} \lambda(\tau) \Delta \tau\right)^{\alpha-1}\right]^{2}(\sigma(r)-t) \Delta t\right)^{\frac{1}{2}} \\
& \times\left(\int_{a}^{\sigma(s)}\left[\psi(\xi)\left(\int_{a}^{\sigma(\xi)} \psi(\tau) \Delta \tau\right)^{\beta-1}\right]^{2}(\sigma(s)-\xi) \Delta \xi\right)^{\frac{1}{2}},
\end{aligned}
$$


holds for all $r, s \in[a, \infty]_{\mathbb{T}}$, with

$$
C(\alpha, \beta, r, s)=\frac{1}{2} \alpha \beta(\sigma(r)-a)^{\frac{1}{2}}(\sigma(s)-a)^{\frac{1}{2}},
$$

and

$$
\begin{aligned}
S_{\alpha, \beta, t, \xi, r, S}= & S\left(\frac{(\sigma(t)-a)\left[\lambda(\zeta)\left(\int_{a}^{\sigma(\zeta)} \lambda(\tau) \Delta \tau\right)^{\alpha-1}\right]^{2}}{\int_{a}^{\sigma(t)}\left[\lambda(\chi)\left(\int_{a}^{\sigma(x)} \lambda(\tau) \Delta \tau\right)^{\alpha-1}\right]^{2} \Delta \chi}\right) \\
& \times S\left(\frac{(\sigma(\tilde{\xi})-a)\left[\psi(\eta)\left(\int_{a}^{\sigma(\eta)} \psi(\tau) \Delta \tau\right)^{\beta-1}\right]^{2}}{\int_{a}^{\sigma(\xi)}\left[\psi(z)\left(\int_{a}^{\sigma(z)} \psi(\tau) \Delta \tau\right)^{\beta-1}\right]^{2} \Delta z}\right) \\
& \times S\left(\frac{(\sigma(r)-a) \int_{a}^{\sigma(t)}\left[\lambda(\xi)\left(\int_{a}^{\sigma(\zeta)} \lambda(\tau) \Delta \tau\right)^{\alpha-1}\right]^{2}}{\int_{a}^{\sigma(r)}\left[\lambda(\chi)\left(\int_{a}^{\sigma(x)} \lambda(\tau) \Delta \tau\right)^{\alpha-1}\right]^{2}(\sigma(r)-\chi) \Delta \chi}\right) \\
& \times S\left(\frac{(\sigma(s)-a) \int_{a}^{\sigma(\xi)}\left[\psi(\eta)\left(\int_{a}^{\sigma(\eta)} \psi(\tau) \Delta \tau\right)^{\beta-1}\right]^{2}}{\int_{a}^{\sigma(s)}\left[\psi(z)\left(\int_{a}^{\sigma(z)} \psi(\tau) \Delta \tau\right)^{\beta-1}\right]^{2}(\sigma(s)-z) \Delta z}\right),
\end{aligned}
$$

such that

$$
\begin{aligned}
& S\left(\frac{(\sigma(t)-a)\left[\lambda(\zeta)\left(\int_{a}^{\sigma(\zeta)} \lambda(\tau) \Delta \tau\right)^{\alpha-1}\right]^{2}}{\int_{a}^{\sigma(t)}\left[\lambda(\chi)\left(\int_{a}^{\sigma(\chi)} \lambda(\tau) \Delta \tau\right)^{\alpha-1}\right]^{2} \Delta \chi}\right) \\
= & \max \left\{S\left(\frac{(\sigma(t)-a)\left[\lambda(a)\left(\int_{a}^{\sigma(a)} \lambda(\tau) \Delta \tau\right)^{\alpha-1}\right]^{2}}{\int_{a}^{\sigma(t)}\left[\lambda(\chi)\left(\int_{a}^{\sigma(\chi)} \lambda(\tau) \Delta \tau\right)^{\alpha-1}\right]^{2} \Delta \chi}\right)\right. \\
& ; S\left(\frac{(\sigma(t)-a)\left[\lambda(t)\left(\int_{a}^{\sigma(t)} \lambda(\tau) \Delta \tau\right)^{\alpha-1}\right]^{2}}{\int_{a}^{\sigma(t)}\left[\lambda(\chi)\left(\int_{a}^{\sigma(\chi)} \lambda(\tau) \Delta \tau\right)^{\alpha-1}\right]^{2} \Delta \chi}\right),
\end{aligned}
$$

and

$$
\begin{aligned}
& S\left(\frac{(\sigma(\xi)-a)\left[\psi(\eta)\left(\int_{a}^{\sigma(\eta)} \psi(\tau) \Delta \tau\right)^{\beta-1}\right]^{2}}{\int_{a}^{\sigma(\xi)}\left[\psi(z)\left(\int_{a}^{\sigma(z)} \psi(\tau) \Delta \tau\right)^{\beta-1}\right]^{2} \Delta z}\right) \\
= & \max \left\{S\left(\frac{(\sigma(\xi)-a)\left[\psi(a)\left(\int_{a}^{\sigma(a)} \psi(\tau) \Delta \tau\right)^{\beta-1}\right]^{2}}{\int_{a}^{\sigma(\xi)}\left[\psi(z)\left(\int_{a}^{\sigma(z)} \psi(\tau) \Delta \tau\right)^{\beta-1}\right]^{2} \Delta z}\right)\right. \\
& \left.; S\left(\frac{(\sigma(\xi)-a)\left[\psi(\xi)\left(\int_{a}^{\sigma(\tilde{\xi})} \psi(\tau) \Delta \tau\right)^{\beta-1}\right]^{2}}{\int_{a}^{\sigma(\xi)}\left[\psi(z)\left(\int_{a}^{\sigma(z)} \psi(\tau) \Delta \tau\right)^{\beta-1}\right]^{2} \Delta z}\right)\right\}
\end{aligned}
$$

Proof. Applying (19) with $\gamma=\alpha$, we have

$$
\left(\int_{a}^{\sigma(t)} \lambda(\tau) \Delta \tau\right)^{\alpha} \geq \alpha \int_{a}^{\sigma(t)} \lambda(\chi)\left(\int_{a}^{\sigma(\chi)} \lambda(\tau) \Delta \tau\right)^{\alpha-1} \Delta \chi .
$$


Multiplying the last inequality by

$$
S\left(\frac{(\sigma(t)-a)\left[\lambda(\zeta)\left(\int_{a}^{\sigma(\zeta)} \lambda(\tau) \Delta \tau\right)^{\alpha-1}\right]^{2}}{\int_{a}^{\sigma(t)}\left[\lambda(\chi)\left(\int_{a}^{\sigma(\chi)} \lambda(\tau) \Delta \tau\right)^{\alpha-1}\right]^{2} \Delta \chi}\right),
$$

we get

$$
\begin{aligned}
& S\left(\frac{(\sigma(t)-a)\left[\lambda(\zeta)\left(\int_{a}^{\sigma(\zeta)} \lambda(\tau) \Delta \tau\right)^{\alpha-1}\right]^{2}}{\int_{a}^{\sigma(t)}\left[\lambda(\chi)\left(\int_{a}^{\sigma(\chi)} \lambda(\tau) \Delta \tau\right)^{\alpha-1}\right]^{2} \Delta \chi}\right)\left(\int_{a}^{\sigma(t)} \lambda(\tau) \Delta \tau\right)^{\alpha} \\
\geq & \alpha \int_{a}^{\sigma(t)} S\left(\frac{(\sigma(t)-a)\left[\lambda(\zeta)\left(\int_{a}^{\sigma(\zeta)} \lambda(\tau) \Delta \tau\right)^{\alpha-1}\right]^{2}}{\int_{a}^{\sigma(t)}\left[\lambda(\chi)\left(\int_{a}^{\sigma(\chi)} \lambda(\tau) \Delta \tau\right)^{\alpha-1}\right]^{2} \Delta \chi}\right) \lambda(\chi)\left(\int_{a}^{\sigma(\chi)} \lambda(\tau) \Delta \tau\right)^{\alpha-1} \Delta \chi .
\end{aligned}
$$

From Lemma (5), the last inequality becomes

$$
\begin{aligned}
& S\left(\frac{(\sigma(t)-a)\left[\lambda(\zeta)\left(\int_{a}^{\sigma(\zeta)} \lambda(\tau) \Delta \tau\right)^{\alpha-1}\right]^{2}}{\int_{a}^{\sigma(t)}\left[\lambda(\chi)\left(\int_{a}^{\sigma(\chi)} \lambda(\tau) \Delta \tau\right)^{\alpha-1}\right]^{2} \Delta \chi}\right)\left(\int_{a}^{\sigma(t)} \lambda(\tau) \Delta \tau\right)^{\alpha} \\
& \geq \alpha \int_{a}^{\sigma(t)} S\left(\frac{(\sigma(t)-a)\left[\lambda(\chi)\left(\int_{a}^{\sigma(x)} \lambda(\tau) \Delta \tau\right)^{\alpha-1}\right]^{2}}{\int_{a}^{\sigma(t)}\left[\lambda(\chi)\left(\int_{a}^{\sigma(\chi)} \lambda(\tau) \Delta \tau\right)^{\alpha-1}\right]^{2} \Delta \chi}\right) \lambda(\chi)\left(\int_{a}^{\sigma(\chi)} \lambda(\tau) \Delta \tau\right)^{\alpha-1} \Delta \chi .
\end{aligned}
$$

Similarly, we have for the decreasing function $\psi$ and $0<\beta \leq 1$ that

$$
\begin{aligned}
& S\left(\frac{(\sigma(\xi)-a)\left[\psi(\eta)\left(\int_{a}^{\sigma(\eta)} \psi(\tau) \Delta \tau\right)^{\beta-1}\right]^{2}}{\int_{a}^{\sigma(\xi)}\left[\psi(z)\left(\int_{a}^{\sigma(z)} \psi(\tau) \Delta \tau\right)^{\beta-1}\right]^{2} \Delta z}\right)\left(\int_{a}^{\sigma(\xi)} \psi(\tau) \Delta \tau\right)^{\beta} \\
& \geq \beta \int_{a}^{\sigma(\xi)} S\left(\frac{(\sigma(\xi)-a)\left[\psi(z)\left(\int_{a}^{\sigma(z)} \psi(\tau) \Delta \tau\right)^{\beta-1}\right]^{2}}{\int_{a}^{\sigma(\xi)}\left[\psi(z)\left(\int_{a}^{\sigma(z)} \psi(\tau) \Delta \tau\right)^{\beta-1}\right]^{2} \Delta z}\right) \psi(z)\left(\int_{a}^{\sigma(z)} \psi(\tau) \Delta \tau\right)^{\beta-1} \Delta z .
\end{aligned}
$$

From (31) and (32), we see that

$$
\begin{aligned}
& S\left(\frac{(\sigma(t)-a)\left[\lambda(\zeta)\left(\int_{a}^{\sigma(\zeta)} \lambda(\tau) \Delta \tau\right)^{\alpha-1}\right]^{2}}{\int_{a}^{\sigma(t)}\left[\lambda(\chi)\left(\int_{a}^{\sigma(\chi)} \lambda(\tau) \Delta \tau\right)^{\alpha-1}\right]^{2} \Delta \chi}\right) S\left(\frac{(\sigma(\xi)-a)\left[\psi(\eta)\left(\int_{a}^{\sigma(\eta)} \psi(\tau) \Delta \tau\right)^{\beta-1}\right]^{2}}{\int_{a}^{\sigma(\xi)}\left[\psi(z)\left(\int_{a}^{\sigma(z)} \psi(\tau) \Delta \tau\right)^{\beta-1}\right]^{2} \Delta z}\right) \\
& \times\left(\int_{a}^{\sigma(t)} \lambda(\tau) \Delta \tau\right)^{\alpha}\left(\int_{a}^{\sigma(\xi)} \psi(\tau) \Delta \tau\right)^{\beta} \\
& \geq \alpha \beta\left(\int_{a}^{\sigma(t)} S\left(\frac{(\sigma(t)-a)\left[\lambda(\chi)\left(\int_{a}^{\sigma(\chi)} \lambda(\tau) \Delta \tau\right)^{\alpha-1}\right]^{2}}{\int_{a}^{\sigma(t)}\left[\lambda(\chi)\left(\int_{a}^{\sigma(x)} \lambda(\tau) \Delta \tau\right)^{\alpha-1}\right]^{2} \Delta \chi}\right) \lambda(\chi)\left(\int_{a}^{\sigma(\chi)} \lambda(\tau) \Delta \tau\right)^{\alpha-1} \times 1 . \Delta \chi\right) \\
& \times\left(\int_{a}^{\sigma(\xi)} S\left(\frac{(\sigma(\xi)-a)\left[\psi(z)\left(\int_{a}^{\sigma(z)} \psi(\tau) \Delta \tau\right)^{\beta-1}\right]^{2}}{\int_{a}^{\sigma(\xi)}\left[\psi(z)\left(\int_{a}^{\sigma(z)} \psi(\tau) \Delta \tau\right)^{\beta-1}\right]^{2} \Delta z}\right) \psi(z)\left(\int_{a}^{\sigma(z)} \psi(\tau) \Delta \tau\right)^{\beta-1} \times 1 . \Delta z\right) .
\end{aligned}
$$


Applying (23) when $\gamma=v=2$, we have

$$
\begin{aligned}
& S\left(\frac{(\sigma(t)-a)\left[\lambda(\zeta)\left(\int_{a}^{\sigma(\zeta)} \lambda(\tau) \Delta \tau\right)^{\alpha-1}\right]^{2}}{\int_{a}^{\sigma(t)}\left[\lambda(\chi)\left(\int_{a}^{\sigma(x)} \lambda(\tau) \Delta \tau\right)^{\alpha-1}\right]^{2} \Delta \chi}\right) S\left(\frac{(\sigma(\xi)-a)\left[\psi(\eta)\left(\int_{a}^{\sigma(\eta)} \psi(\tau) \Delta \tau\right)^{\beta-1}\right]^{2}}{\int_{a}^{\sigma(\xi)}\left[\psi(z)\left(\int_{a}^{\sigma(z)} \psi(\tau) \Delta \tau\right)^{\beta-1}\right]^{2} \Delta z}\right) \\
& \times\left(\int_{a}^{\sigma(t)} \lambda(\tau) \Delta \tau\right)^{\alpha}\left(\int_{a}^{\sigma(\xi)} \psi(\tau) \Delta \tau\right)^{\beta} \\
& \geq \alpha \beta(\sigma(t)-a)^{\frac{1}{2}}\left(\int_{a}^{\sigma(t)}\left[\lambda(\chi)\left(\int_{a}^{\sigma(\chi)} \lambda(\tau) \Delta \tau\right)^{\alpha-1}\right]^{2} \Delta \chi\right)^{\frac{1}{2}} \\
& \times(\sigma(\xi)-a)^{\frac{1}{2}}\left(\int_{a}^{\sigma(\xi)}\left[\psi(z)\left(\int_{a}^{\sigma(z)} \psi(\tau) \Delta \tau\right)^{\beta-1}\right]^{2} \Delta z\right)^{\frac{1}{2}} .
\end{aligned}
$$

Multiplying (34) by

$$
S\left(\frac{(\sigma(r)-a) \int_{a}^{\sigma(t)}\left[\lambda(\zeta)\left(\int_{a}^{\sigma(\zeta)} \lambda(\tau) \Delta \tau\right)^{\alpha-1}\right]^{2}}{\int_{a}^{\sigma(r)}\left[\lambda(\chi)\left(\int_{a}^{\sigma(\chi)} \lambda(\tau) \Delta \tau\right)^{\alpha-1}\right]^{2}(\sigma(r)-\chi) \Delta \chi}\right) S\left(\frac{(\sigma(s)-a) \int_{a}^{\sigma(\xi)}\left[\psi(\eta)\left(\int_{a}^{\sigma(\eta)} \psi(\tau) \Delta \tau\right)^{\beta-1}\right]^{2}}{\int_{a}^{\sigma(s)}\left[\psi(z)\left(\int_{a}^{\sigma(z)} \psi(\tau) \Delta \tau\right)^{\beta-1}\right]^{2}(\sigma(s)-z) \Delta z}\right),
$$

we see that

$$
\begin{aligned}
& S_{\alpha, \beta, t, \xi, \xi, s}\left(\int_{a}^{\sigma(t)} \lambda(\tau) \Delta \tau\right)^{\alpha} \\
& =\left(\int_{a}^{\sigma(t)} \lambda(\tau) \Delta \tau\right)^{\alpha} S\left(\frac{(\sigma(r)-a) \int_{a}^{\sigma(t)}\left[\lambda(\zeta)\left(\int_{a}^{\sigma(\zeta)} \lambda(\tau) \Delta \tau\right)^{\alpha-1}\right]^{2}}{\int_{a}^{\sigma(r)}\left[\lambda(\chi)\left(\int_{a}^{\sigma(x)} \lambda(\tau) \Delta \tau\right)^{\alpha-1}\right]^{2}(\sigma(r)-\chi) \Delta \chi}\right) \\
& \times\left(\int_{a}^{\sigma(\xi)} \psi(\tau) \Delta \tau\right)^{\beta} S\left(\frac{(\sigma(s)-a) \int_{a}^{\sigma(\xi)}\left[\psi(\eta)\left(\int_{a}^{\sigma(\eta)} \psi(\tau) \Delta \tau\right)^{\beta-1}\right]^{2}}{\int_{a}^{\sigma(s)}\left[\psi(z)\left(\int_{a}^{\sigma(z)} \psi(\tau) \Delta \tau\right)^{\beta-1}\right]^{2}(\sigma(s)-z) \Delta z}\right) \\
& \times S\left(\frac{(\sigma(t)-a)\left[\lambda(\zeta)\left(\int_{a}^{\sigma(\zeta)} \lambda(\tau) \Delta \tau\right)^{\alpha-1}\right]^{2}}{\int_{a}^{\sigma(t)}\left[\lambda(\chi)\left(\int_{a}^{\sigma(\chi)} \lambda(\tau) \Delta \tau\right)^{\alpha-1}\right]^{2} \Delta \chi}\right) S\left(\frac{(\sigma(\xi)-a)\left[\psi(\eta)\left(\int_{a}^{\sigma(\eta)} \psi(\tau) \Delta \tau\right)^{\beta-1}\right]^{2}}{\int_{a}^{\sigma(\xi)}\left[\psi(z)\left(\int_{a}^{\sigma(z)} \psi(\tau) \Delta \tau\right)^{\beta-1}\right]^{2} \Delta z}\right) \\
& \geq \alpha \beta(\sigma(t)-a)^{\frac{1}{2}} S\left(\frac{(\sigma(r)-a) \int_{a}^{\sigma(t)}\left[\lambda(\zeta)\left(\int_{a}^{\sigma(\zeta)} \lambda(\tau) \Delta \tau\right)^{\alpha-1}\right]^{2}}{\int_{a}^{\sigma(r)}\left[\lambda(\chi)\left(\int_{a}^{\sigma(\chi)} \lambda(\tau) \Delta \tau\right)^{\alpha-1}\right]^{2}(\sigma(r)-\chi) \Delta \chi}\right) \\
& \times(\sigma(\xi)-a)^{\frac{1}{2}} S\left(\frac{(\sigma(s)-a) \int_{a}^{\sigma(\xi)}\left[\psi(\eta)\left(\int_{a}^{\sigma(\eta)} \psi(\tau) \Delta \tau\right)^{\beta-1}\right]^{2}}{\int_{a}^{\sigma(s)}\left[\psi(z)\left(\int_{a}^{\sigma(z)} \psi(\tau) \Delta \tau\right)^{\beta-1}\right]^{2}(\sigma(s)-z) \Delta z}\right) \\
& \times\left(\begin{array}{c}
\left(\int_{a}^{\sigma(t)}\left[\lambda(\chi)\left(\int_{a}^{\sigma(\chi)} \lambda(\tau) \Delta \tau\right)^{\alpha-1}\right]^{2} \Delta \chi\right. \\
\cdot\left(\int_{a}^{\sigma(\xi)}\left[\psi(z)\left(\int_{a}^{\sigma(z)} \psi(\tau) \Delta \tau\right)^{\beta-1}\right]^{2} \Delta z\right)
\end{array}\right)
\end{aligned}
$$


Dividing the two sides of (35) by $(\sigma(t)-a)^{\frac{1}{2}}(\sigma(\xi)-a)^{\frac{1}{2}}$ and then taking the integration over $t$ from $a$ to $\sigma(r)$ and the integration over $\xi$ from $a$ to $\sigma(s)$, we get

$$
\begin{aligned}
& \int_{a}^{\sigma(s)} \int_{a}^{\sigma(r)} \frac{S_{\alpha, \beta, t, \xi, r, s}\left(\int_{a}^{\sigma(t)} \lambda(\tau) \Delta \tau\right)^{\alpha}\left(\int_{a}^{\sigma(\xi)} \psi(\tau) \Delta \tau\right)^{\beta}}{(\sigma(t)-a)^{\frac{1}{2}}(\sigma(\xi)-a)^{\frac{1}{2}}} \Delta t \Delta \xi \\
& \geq \alpha \beta \int_{a}^{\sigma(r)} S\left(\frac{(\sigma(r)-a) \int_{a}^{\sigma(t)}\left[\lambda(\zeta)\left(\int_{a}^{\sigma(\zeta)} \lambda(\tau) \Delta \tau\right)^{\alpha-1}\right]^{2}}{\int_{a}^{\sigma(r)}\left[\lambda(\chi)\left(\int_{a}^{\sigma(\chi)} \lambda(\tau) \Delta \tau\right)^{\alpha-1}\right]^{2}(\sigma(r)-\chi) \Delta \chi}\right) \\
& \times\left(\int_{a}^{\sigma(t)}\left[\lambda(\chi)\left(\int_{a}^{\sigma(\chi)} \lambda(\tau) \Delta \tau\right)^{\alpha-1}\right]^{2} \Delta \chi\right)^{\frac{1}{2}} \Delta t \\
& \left.\left.\times \int_{a}^{\sigma(s)} S\left(\frac{(\sigma(s)-a) \int_{a}^{\sigma(\xi)}\left[\psi(\eta)\left(\int_{a}^{\sigma(\eta)} \psi(\tau) \Delta \tau\right)^{\beta-1}\right]^{2}}{\int_{a}^{\sigma(s)}\left[\psi(z)\left(\int_{a}^{\sigma(z)} \psi(\tau) \Delta \tau\right)^{\beta-1}\right]^{2}(\sigma(s)-z) \Delta z}\right)^{\beta-1}\right]^{2} \Delta z\right)^{\frac{1}{2}} \Delta \xi
\end{aligned}
$$

By applying (16) on the term

$$
\int_{a}^{\sigma(r)}\left[\lambda(\chi)\left(\int_{a}^{\sigma(\chi)} \lambda(\tau) \Delta \tau\right)^{\alpha-1}\right]^{2}(\sigma(r)-\chi) \Delta \chi
$$

with $u(\chi)=(\sigma(r)-\chi)$ and $v^{\Delta}(\chi)=\left[\lambda(\chi)\left(\int_{a}^{\sigma(\chi)} \lambda(\tau) \Delta \tau\right)^{\alpha-1}\right]^{2}$, we get

$$
\begin{aligned}
& \int_{a}^{\sigma(r)}\left[\lambda(\chi)\left(\int_{a}^{\sigma(\chi)} \lambda(\tau) \Delta \tau\right)^{\alpha-1}\right]^{2}(\sigma(r)-\chi) \Delta \chi \\
= & \left.(\sigma(r)-\chi) v(\chi)\right|_{a} ^{\sigma(r)}+\int_{a}^{\sigma(r)} v^{\sigma}(\chi) \Delta \chi,
\end{aligned}
$$

where $v(\chi)=\int_{a}^{\chi}\left[\lambda(\theta)\left(\int_{a}^{\sigma(\theta)} \lambda(\tau) \Delta \tau\right)^{\alpha-1}\right]^{2} \Delta \theta$ and then (where $v(a)=0$ )

$$
\begin{aligned}
& \int_{a}^{\sigma(r)}\left[\lambda(\chi)\left(\int_{a}^{\sigma(\chi)} \lambda(\tau) \Delta \tau\right)^{\alpha-1}\right]^{2}(\sigma(r)-\chi) \Delta \chi \\
= & \int_{a}^{\sigma(r)} \int_{a}^{\sigma(\chi)}\left[\lambda(\theta)\left(\int_{a}^{\sigma(\theta)} \lambda(\tau) \Delta \tau\right)^{\alpha-1}\right]^{2} \Delta \theta \Delta \chi .
\end{aligned}
$$

Similarly, we see that

$$
\begin{aligned}
& \int_{a}^{\sigma(s)}\left[\psi(z)\left(\int_{a}^{\sigma(z)} \psi(\tau) \Delta \tau\right)^{\beta-1}\right]^{2}(\sigma(s)-z) \Delta z \\
= & \int_{a}^{\sigma(s)} \int_{a}^{\sigma(z)}\left[\psi(\theta)\left(\int_{a}^{\sigma(\theta)} \psi(\tau) \Delta \tau\right)^{\beta-1}\right]^{2} \Delta \theta \Delta z .
\end{aligned}
$$


Substituting (37) and (38) into (36) and then by applying (23) when $\gamma=v=2$, we observe that

$$
\begin{aligned}
& \int_{a}^{\sigma(s)} \int_{a}^{\sigma(r)} \frac{S_{\alpha, \beta, t, \xi, r, s}\left(\int_{a}^{\sigma(t)} \lambda(\tau) \Delta \tau\right)^{\alpha}\left(\int_{a}^{\sigma(\xi)} \psi(\tau) \Delta \tau\right)^{\beta}}{(\sigma(t)-a)^{\frac{1}{2}}(\sigma(\xi)-a)^{\frac{1}{2}}} \Delta t \Delta \xi \\
& \geq \alpha \beta \int_{a}^{\sigma(r)} S\left(\frac{(\sigma(r)-a) \int_{a}^{\sigma(t)}\left[\lambda(\zeta)\left(\int_{a}^{\sigma(\zeta)} \lambda(\tau) \Delta \tau\right)^{\alpha-1}\right]^{2} \Delta \zeta}{\int_{a}^{\sigma(r)} \int_{a}^{\sigma(\chi)}\left[\lambda(\theta)\left(\int_{a}^{\sigma(\theta)} \lambda(\tau) \Delta \tau\right)^{\alpha-1}\right]^{2} \Delta \theta \Delta \chi}\right) \\
& \times\left(\int_{a}^{\sigma(t)}\left[\lambda(\chi)\left(\int_{a}^{\sigma(\chi)} \lambda(\tau) \Delta \tau\right)^{\alpha-1}\right]^{2} \Delta \chi\right)^{\frac{1}{2}} \times 1 \Delta t \\
& \times \int_{a}^{\sigma(s)} S\left[\frac{(\sigma(s)-a) \int_{a}^{\sigma(\xi)}\left[\psi(\eta)\left(\int_{a}^{\sigma(\eta)} \psi(\tau) \Delta \tau\right)^{\beta-1}\right]^{2} \Delta \eta}{\int_{a}^{\sigma(s)} \int_{a}^{\sigma(z)}\left[\psi(\theta)\left(\int_{a}^{\sigma(\theta)} \psi(\tau) \Delta \tau\right)^{\beta-1}\right]^{2} \Delta \theta \Delta z}\right)^{\frac{1}{2}} \times 1 \Delta \xi \\
& \left.\left.\times\left(\int_{a}^{\sigma(\xi)}\left[\psi(z)\left(\int_{a}^{\sigma(z)} \psi(\tau) \Delta \tau\right)^{\beta-1} \Delta z\right)^{2} \times 1\right]^{\frac{1}{2}}\right]^{\frac{1}{2}} \Delta z \Delta \xi\right)^{\frac{1}{2}} . \\
& \geq \alpha \beta(\sigma(r)-a)^{\frac{1}{2}}\left(\int _ { a } ^ { \sigma ( r ) } \int _ { a } ^ { \sigma ( t ) } \left[\lambda(\chi)\left(\int_{a}^{\sigma(\chi)} \lambda(\tau) \Delta \tau\right)^{\alpha-1} \Delta \chi \Delta t\right.\right. \\
& \times(\sigma(s)-a)^{\frac{1}{2}}\left(\int _ { a } ^ { \sigma ( s ) } \int _ { a } ^ { \sigma ( \xi ) } \left[\psi(z)\left(\int_{a}^{\sigma(z)} \psi(\tau) \Delta \tau\right)^{2}\right.\right.
\end{aligned}
$$

From (37)-(39), the last inequality becomes

$$
\begin{aligned}
& \int_{a}^{\sigma(s)} \int_{a}^{\sigma(r)} \frac{S_{\alpha, \beta, t, \xi, r, s}\left(\int_{a}^{\sigma(t)} \lambda(\tau) \Delta \tau\right)^{\alpha}\left(\int_{a}^{\sigma(\xi)} \psi(\tau) \Delta \tau\right)^{\beta}}{(\sigma(t)-a)^{\frac{1}{2}}(\sigma(\xi)-a)^{\frac{1}{2}}} \Delta t \Delta \xi \\
\geq & \alpha \beta(\sigma(r)-a)^{\frac{1}{2}}\left(\int_{a}^{\sigma(r)}\left[\lambda(t)\left(\int_{a}^{\sigma(t)} \lambda(\tau) \Delta \tau\right)^{\alpha-1}\right]^{2}(\sigma(r)-t) \Delta t\right)^{\frac{1}{2}} \\
& \times(\sigma(s)-a)^{\frac{1}{2}}\left(\int_{a}^{\sigma(s)}\left[\psi(\xi)\left(\int_{a}^{\sigma(\xi)} \psi(\tau) \Delta \tau\right)^{\beta-1}\right]^{2}(\sigma(s)-\xi) \Delta \xi\right)^{\frac{1}{2}} \\
= & 2 C(\alpha, \beta, r, s)\left(\int_{a}^{\sigma(r)}\left[\lambda(t)\left(\int_{a}^{\sigma(t)} \lambda(\tau) \Delta \tau\right)^{\alpha-1}\right]^{2}(\sigma(r)-t) \Delta t\right)^{\frac{1}{2}} \\
& \times\left(\int_{a}^{\sigma(s)}\left[\psi(\xi)\left(\int_{a}^{\sigma(\xi)} \psi(\tau) \Delta \tau\right)^{\beta-1}\right]^{2}(\sigma(s)-\xi) \Delta \xi\right)^{\frac{1}{2}},
\end{aligned}
$$

which is (29).

Remark 1. As a special case of Theorem 2, when $\mathbb{T}=\mathbb{N}$, we can get (9) proved by Zhao and Cheung [12]. 
Theorem 3. Let $a \in \mathbb{T}$ and $\lambda, \omega$ be nonnegative functions. In addition, we assume that $\alpha, \beta$ are positive functions. If $\phi, \psi$ are nonnegative, concave, and supermultiplicative functions, then the inequality

$$
\begin{aligned}
& \int_{a}^{\sigma(s)} \int_{a}^{\sigma(r)} \frac{S_{t, r, s, \zeta} \phi(\Lambda(t)) \psi(\Omega(\zeta))}{(\sigma(t)-a)^{\frac{1}{2}}(\sigma(\zeta)-a)^{\frac{1}{2}}} \Delta t \Delta \zeta \\
\geq & 2 M(r, s)\left(\int_{a}^{\sigma(r)}\left[\alpha(\chi) \phi\left(\frac{\lambda(\chi)}{\alpha(\chi)}\right)\right]^{2}(\sigma(r)-\chi) \Delta \chi\right)^{\frac{1}{2}} \\
& \times\left(\int_{a}^{\sigma(s)}\left[\beta(z) \psi\left(\frac{\omega(z)}{\beta(z)}\right)\right]^{2}(\sigma(s)-z) \Delta z\right)^{\frac{1}{2}},
\end{aligned}
$$

holds for all $r, s \in[a, \infty]_{\mathbb{T}}$, with

$$
\begin{aligned}
& M(r, s)=\frac{1}{2}\left(\int_{a}^{\sigma(r)}\left(\frac{\phi(\Phi(t))}{\Phi(t)}\right)^{2} \Delta t\right)^{\frac{1}{2}}\left(\int_{a}^{\sigma(s)}\left(\frac{\psi(\Psi(\zeta))}{\Psi(\zeta)}\right)^{2} \Delta \zeta\right)^{\frac{1}{2}}, \\
& S_{t, r, s, \zeta}=S\left(\frac{\left(\int_{a}^{\sigma(r)}\left[\alpha(\chi) \phi\left(\frac{\lambda(\chi)}{\alpha(\chi)}\right)\right]^{2}(\sigma(r)-\chi) \Delta \chi\right)\left(\frac{\phi(\Phi(t))}{\Phi(t)}\right)^{2}}{\left(\int_{a}^{\sigma(r)}\left(\frac{\phi(\Phi(t))}{\Phi(t)}\right)^{2} \Delta t\right)\left(\int_{a}^{\sigma(t)}\left[\alpha(\chi) \phi\left(\frac{\lambda(\chi)}{\alpha(\chi)}\right)\right]^{2} \Delta \chi\right)}\right) \\
& \times S\left(\frac{\left(\int_{a}^{\sigma(s)}\left[\beta(z) \psi\left(\frac{\omega(z)}{\beta(z)}\right)\right]^{2}(\sigma(s)-z) \Delta z\right)\left(\frac{\psi(\Psi(\zeta))}{\Psi(\zeta)}\right)^{2}}{\left(\int_{a}^{\sigma(s)}\left(\frac{\psi(\Psi(\zeta))}{\Psi(\zeta)}\right)^{2} \Delta \zeta\right)\left(\int_{a}^{\sigma(\zeta)}\left[\beta(z) \psi\left(\frac{\omega(z)}{\beta(z)}\right)\right]^{2} \Delta z\right)}\right), \\
& \Lambda(t)=\int_{a}^{\sigma(t)} S\left(\frac{(\sigma(t)-a)\left[\alpha(\chi) \phi\left(\frac{\lambda(\chi)}{\alpha(\chi)}\right)\right]^{2}}{\int_{a}^{\sigma(t)}\left[\alpha(\chi) \phi\left(\frac{\lambda(\chi)}{\alpha(\chi)}\right)\right]^{2} \Delta \chi}\right) \lambda(\chi) \Delta \chi \\
& \Omega(\zeta)=\int_{a}^{\sigma(\zeta)} S\left(\frac{(\sigma(\zeta)-a)\left[\beta(z) \psi\left(\frac{\omega(z)}{\beta(z)}\right)\right]^{2}}{\int_{a}^{\sigma(\zeta)}\left[\beta(z) \psi\left(\frac{\omega(z)}{\beta(z)}\right)\right]^{2} \Delta z}\right) \omega(z) \Delta z \\
& \Phi(t)=\int_{a}^{\sigma(t)} S\left(\frac{(\sigma(t)-a)\left[\alpha(\chi) \phi\left(\frac{\lambda(\chi)}{\alpha(\chi)}\right)\right]^{2}}{\int_{a}^{\sigma(t)}\left[\alpha(\chi) \phi\left(\frac{\lambda(\chi)}{\alpha(\chi)}\right)\right]^{2} \Delta \chi}\right) \alpha(\chi) \Delta \chi,
\end{aligned}
$$

and

$$
\Psi(\zeta)=\int_{a}^{\sigma(\zeta)} S\left(\frac{(\sigma(\zeta)-a)\left[\beta(z) \psi\left(\frac{\omega(z)}{\beta(z)}\right)\right]^{2}}{\int_{a}^{\sigma(\zeta)}\left[\beta(z) \psi\left(\frac{\omega(z)}{\beta(z)}\right)\right]^{2} \Delta z}\right) \beta(z) \Delta z
$$


Proof. Using the fact that $\phi$ is a supermultiplicative function, applying Jensen's inequality and then applying (23) with $\gamma=v=2$. Then,

$$
\begin{aligned}
& \phi(\Lambda(t))=\phi\left(\frac{\Phi(t) \int_{a}^{\sigma(t)} S\left(\frac{(\sigma(t)-a)\left[\alpha(\chi) \phi\left(\frac{\lambda(\chi)}{\alpha(\chi)}\right)\right]^{2}}{\int_{a}^{\sigma(t)}\left[\alpha(\chi) \phi\left(\frac{\lambda(\chi)}{\alpha(\chi)}\right)\right]^{2} \Delta \chi}\right) \alpha(\chi) \lambda(\chi) / \alpha(\chi) \Delta \chi}{\int_{a}^{\sigma(t)} S\left(\frac{(\sigma(t)-a)\left[\alpha(\chi) \phi\left(\frac{\lambda(\chi)}{\alpha(x)}\right)\right]^{2}}{\int_{a}^{\sigma(t)}\left[\alpha(\chi) \phi\left(\frac{\lambda(\chi)}{\alpha(\chi)}\right)\right]^{2} \Delta \chi}\right) \alpha(\chi) \Delta \chi}\right) \\
& \geq \phi(\Phi(t)) \phi\left(\frac{\int_{a}^{\sigma(t)} S\left(\frac{(\sigma(t)-a)\left[\alpha(\chi) \phi\left(\frac{\lambda(\chi)}{\alpha(\chi)}\right)\right]^{2}}{\int_{a}^{\sigma(t)}\left[\alpha(\chi) \phi\left(\frac{\lambda(\chi)}{\alpha(x)}\right)\right]^{2} \Delta \chi}\right) \alpha(\chi)\left[\frac{\lambda(\chi)}{\alpha(\chi)}\right] \Delta \chi}{\int_{a}^{\sigma(t)} S\left(\frac{(\sigma(t)-a)\left[\alpha(\chi) \phi\left(\frac{\lambda(\chi)}{\alpha(\chi)}\right)\right]^{2}}{\int_{a}^{\sigma(t)}\left[\alpha(\chi) \phi\left(\frac{\lambda(x)}{\alpha(\chi)}\right)\right]^{2} \Delta \chi}\right) \alpha(\chi) \Delta \chi}\right) \\
& \geq \frac{\phi(\Phi(t))}{\Phi(t)} \int_{a}^{\sigma(t)} S\left(\frac{(\sigma(t)-a)\left[\alpha(\chi) \phi\left(\frac{\lambda(\chi)}{\alpha(\chi)}\right)\right]^{2}}{\int_{a}^{\sigma(t)}\left[\alpha(\chi) \phi\left(\frac{\lambda(\chi)}{\alpha(\chi)}\right)\right]^{2} \Delta \chi}\right) \alpha(\chi) \phi\left[\frac{\lambda(\chi)}{\alpha(\chi)}\right] \times 1 \Delta \chi \\
& \geq \frac{\phi(\Phi(t))}{\Phi(t)}(\sigma(t)-a)^{\frac{1}{2}}\left(\int_{a}^{\sigma(t)}\left[\alpha(\chi) \phi\left(\frac{\lambda(\chi)}{\alpha(\chi)}\right)\right]^{2} \Delta \chi\right)^{\frac{1}{2}} \text {. }
\end{aligned}
$$

Similarly, we can get

$$
\psi(\Omega(\zeta)) \geq \frac{\psi(\Psi(\zeta))}{\Psi(\zeta)}(\sigma(\zeta)-a)^{\frac{1}{2}}\left(\int_{a}^{\sigma(\zeta)}\left[\beta(z) \psi\left(\frac{\omega(z)}{\beta(z)}\right)\right]^{2} \Delta z\right)^{\frac{1}{2}}
$$

Multiplying the both sides of (41) and (42), respectively, by

$$
S\left(\frac{\left(\int_{a}^{\sigma(r)}\left[\alpha(\chi) \phi\left(\frac{\lambda(\chi)}{\alpha(\chi)}\right)\right]^{2}(\sigma(r)-\chi) \Delta \chi\right)\left(\frac{\phi(\Phi(t))}{\Phi(t)}\right)^{2}}{\left(\int_{a}^{\sigma(r)}\left(\frac{\phi(\Phi(t))}{\Phi(t)}\right)^{2} \Delta t\right)\left(\int_{a}^{\sigma(t)}\left[\alpha(\chi) \phi\left(\frac{\lambda(\chi)}{\alpha(\chi)}\right)\right]^{2} \Delta \chi\right)}\right)
$$

and

$$
S\left(\frac{\left(\int_{a}^{\sigma(s)}\left[\beta(z) \psi\left(\frac{\omega(z)}{\beta(z)}\right)\right]^{2}(\sigma(s)-z) \Delta z\right)\left(\frac{\psi(\Psi(\zeta))}{\Psi(\zeta)}\right)^{2}}{\left(\int_{a}^{\sigma(s)}\left(\frac{\psi(\Psi(\zeta))}{\Psi(\zeta)}\right)^{2} \Delta \zeta\right)\left(\int_{a}^{\sigma(\zeta)}\left[\beta(z) \psi\left(\frac{\omega(z)}{\beta(z)}\right)\right]^{2} \Delta z\right)}\right)
$$


and then multiplying these inequalities, we get

$$
\begin{aligned}
& S\left(\frac{\left(\int_{a}^{\sigma(s)}\left[\beta(z) \psi\left(\frac{\omega(z)}{\beta(z)}\right)\right]^{2}(\sigma(s)-z) \Delta z\right)\left(\frac{\psi(\Psi(\zeta))}{\Psi(\zeta)}\right)^{2}}{\left(\int_{a}^{\sigma(s)}\left(\frac{\psi(\Psi(\zeta))}{\Psi(\zeta)}\right)^{2} \Delta \zeta\right)\left(\int_{a}^{\sigma(\zeta)}\left[\beta(z) \psi\left(\frac{\omega(z)}{\beta(z)}\right)\right]^{2} \Delta z\right)}\right) \phi(\Lambda(t)) \psi(\Omega(\zeta)) \\
& \times S\left(\frac{\left(\int_{a}^{\sigma(r)}\left[\alpha(\chi) \phi\left(\frac{\lambda(\chi)}{\alpha(\chi)}\right)\right]^{2}(\sigma(r)-\chi) \Delta \chi\right)\left(\frac{\phi(\Phi(t))}{\Phi(t)}\right)^{2}}{\left(\int_{a}^{\sigma(r)}\left(\frac{\phi(\Phi(t))}{\Phi(t)}\right)^{2} \Delta t\right)\left(\int_{a}^{\sigma(t)}\left[\alpha(\chi) \phi\left(\frac{\lambda(\chi)}{\alpha(\chi)}\right)\right]^{2} \Delta \chi\right)}\right) \\
\geq & \frac{\phi(\Phi(t))}{\Phi(t)}(\sigma(t)-a)^{\frac{1}{2}}\left(\int_{a}^{\sigma(t)}\left[\alpha(\chi) \phi\left(\frac{\lambda(\chi)}{\alpha(\chi)}\right)\right]^{2} \Delta \chi\right)^{\frac{1}{2}} \\
& \times S\left(\frac{\left(\int_{a}^{\sigma(r)}\left[\alpha(\chi) \phi\left(\frac{\lambda(\chi)}{\alpha(\chi)}\right)\right]^{2}(\sigma(r)-\chi) \Delta \chi\right)\left(\frac{\phi(\Phi(t))}{\Phi(t)}\right)^{2}}{\left(\int_{a}^{\sigma(r)}\left(\frac{\phi(\Phi(t))}{\Phi(t)}\right)^{2} \Delta t\right)\left(\int_{a}^{\sigma(t)}\left[\alpha(\chi) \phi\left(\frac{\lambda(\chi)}{\alpha(\chi)}\right)\right]^{2} \Delta \chi\right)}\right) \\
& \times \frac{\psi(\Psi(\zeta))}{\Psi(\zeta)}(\sigma(\zeta)-a)^{\frac{1}{2}}\left(\int_{a}^{\sigma(\zeta)}\left[\beta(z) \psi\left(\frac{\omega(z)}{\beta(z)}\right)\right]^{2} \Delta z\right)^{\frac{1}{2}} \\
& \times S\left(\frac{\left(\int_{a}^{\sigma(s)}\left[\beta(z) \psi\left(\frac{\omega(z)}{\beta(z)}\right)\right]^{2}(\sigma(s)-z) \Delta z\right)\left(\frac{\psi(\Psi(\zeta))}{\Psi(\zeta)}\right)^{2}}{\left(\int_{a}^{\sigma(s)}\left(\frac{\psi(\Psi(\zeta))}{\Psi(\zeta)}\right)^{2} \Delta \zeta\right)\left(\int_{a}^{\sigma(\zeta)}\left[\beta(z) \psi\left(\frac{\omega(z)}{\beta(z)}\right)\right]^{2} \Delta z\right)}\right)
\end{aligned}
$$

By dividing the two sides of $(43)$ on $(\sigma(t)-a)^{\frac{1}{2}}(\sigma(\zeta)-a)^{\frac{1}{2}}$ and then taking the integration over $\zeta$ from $a$ to $\sigma(s)$ and then the integration over $t$ from $a$ to $\sigma(r)$, we observe that

$$
\begin{aligned}
& \int_{a}^{\sigma(s)} \int_{a}^{\sigma(r)} \frac{S_{t, r, s, \zeta} \phi(\Lambda(t)) \psi(\Omega(\zeta))}{(\sigma(t)-a)^{\frac{1}{2}}(\sigma(\zeta)-a)^{\frac{1}{2}}} \Delta t \Delta \zeta \\
\geq & \int_{a}^{\sigma(r)} S\left(\frac{\left(\int_{a}^{\sigma(r)}\left[\alpha(\chi) \phi\left(\frac{\lambda(\chi)}{\alpha(\chi)}\right)\right]^{2}(\sigma(r)-\chi) \Delta \chi\right)\left(\frac{\phi(\Phi(t))}{\Phi(t)}\right)^{2}}{\left(\int_{a}^{\sigma(r)}\left(\frac{\phi(\Phi(t))}{\Phi(t)}\right)^{2} \Delta t\right)\left(\int_{a}^{\sigma(t)}\left[\alpha(\chi) \phi\left(\frac{\lambda(\chi)}{\alpha(\chi)}\right)\right]^{2} \Delta \chi\right)}\right) \\
\times & \frac{\phi(\Phi(t))}{\Phi(t)}\left(\int_{a}^{\sigma(t)}\left[\alpha(\chi) \phi\left(\frac{\lambda(\chi)}{\alpha(\chi)}\right)\right]^{2} \Delta \chi\right)^{\frac{1}{2}} \Delta t \\
\times & \int_{a}^{\sigma(s)} S\left(\frac{\left(\int_{a}^{\sigma(s)}\left[\beta(z) \psi\left(\frac{\omega(z)}{\beta(z)}\right)\right]^{2}(\sigma(s)-z) \Delta z\right)\left(\frac{\psi(\Psi(\zeta))}{\Psi(\zeta)}\right)^{2}}{\left(\int_{a}^{\sigma(s)}\left(\frac{\psi(\Psi(\zeta))}{\Psi(\zeta)}\right)^{2} \Delta \zeta\right)\left(\int_{a}^{\sigma(\zeta)}\left[\beta(z) \psi\left(\frac{\omega(z)}{\beta(z)}\right)\right]^{2} \Delta z\right)}\right) \\
\times & \frac{\psi(\Psi(\zeta))}{\Psi(\zeta)}\left(\int_{a}^{\sigma(\zeta)}\left[\beta(z) \psi\left(\frac{\omega(z)}{\beta(z)}\right)\right]^{2} \Delta z\right)^{\frac{1}{2}} \Delta \zeta .
\end{aligned}
$$

By using (16), we can see that

$$
\begin{aligned}
& \int_{a}^{\sigma(r)}\left[\alpha(\chi) \phi\left(\frac{\lambda(\chi)}{\alpha(\chi)}\right)\right]^{2}(\sigma(r)-\chi) \Delta \chi \\
= & \int_{a}^{\sigma(r)} \int_{a}^{\sigma(\chi)}\left[\alpha(\theta) \phi\left(\frac{\lambda(\theta)}{\alpha(\theta)}\right)\right]^{2} \Delta \theta \Delta \chi .
\end{aligned}
$$


In addition, we can obtain that

$$
\begin{aligned}
& \int_{a}^{\sigma(s)}\left[\beta(z) \psi\left(\frac{\omega(z)}{\beta(z)}\right)\right]^{2}(\sigma(s)-z) \Delta z \\
= & \int_{a}^{\sigma(s)} \int_{a}^{\sigma(z)}\left[\beta(\theta) \psi\left(\frac{\omega(\theta)}{\beta(\theta)}\right)\right]^{2} \Delta \theta \Delta z .
\end{aligned}
$$

Substituting (45) and (46) into (44), we have

$$
\begin{aligned}
& \int_{a}^{\sigma(s)} \int_{a}^{\sigma(r)} \frac{S_{t, r, s, \zeta} \phi(\Lambda(t)) \psi(\Omega(\zeta))}{(\sigma(t)-a)^{\frac{1}{2}}(\sigma(\zeta)-a)^{\frac{1}{2}}} \Delta t \Delta \zeta \\
\geq & \int_{a}^{\sigma(r)} S\left(\frac{\left(\int_{a}^{\sigma(r)} \int_{a}^{\sigma(\chi)}\left[\alpha(\theta) \phi\left(\frac{\lambda(\theta)}{\alpha(\theta)}\right)\right]^{2} \Delta \theta \Delta \chi\right)\left(\frac{\phi(\Phi(t))}{\Phi(t)}\right)^{2}}{\left(\int_{a}^{\sigma(r)}\left(\frac{\phi(\Phi(t))}{\Phi(t)}\right)^{2} \Delta t\right)\left(\int_{a}^{\sigma(t)}\left[\alpha(\chi) \phi\left(\frac{\lambda(\chi)}{\alpha(\chi)}\right)\right]^{2} \Delta \chi\right)}\right) \\
\times & \frac{\phi(\Phi(t))}{\Phi(t)}\left(\int_{a}^{\sigma(t)}\left[\alpha(\chi) \phi\left(\frac{\lambda(\chi)}{\alpha(\chi)}\right)\right]^{2} \Delta \chi\right)^{\frac{1}{2}} \Delta t \\
\times & \int_{a}^{\sigma(s)} S\left(\frac{\left(\int_{a}^{\sigma(s)} \int_{a}^{\sigma(z)}\left[\beta(\theta) \psi\left(\frac{\omega(\theta)}{\beta(\theta)}\right)\right]^{2} \Delta \theta \Delta z\right)\left(\frac{\psi(\Psi(\zeta))}{\Psi(\zeta)}\right)^{2}}{\left(\int_{a}^{\sigma(s)}\left(\frac{\psi(\Psi(\zeta))}{\Psi(\zeta)}\right)^{2} \Delta \zeta\right)\left(\int_{a}^{\sigma(\zeta)}\left[\beta(z) \psi\left(\frac{\omega(z)}{\beta(z)}\right)\right]^{2} \Delta z\right)}\right) \\
\times & \frac{\psi(\Psi(\zeta))}{\Psi(\zeta)}\left(\int_{a}^{\sigma(\zeta)}\left[\beta(z) \psi\left(\frac{\omega(z)}{\beta(z)}\right)\right]^{2} \Delta z\right)^{\frac{1}{2}} \Delta \zeta .
\end{aligned}
$$

Applying (23) with $\gamma=v=2$ on the right-hand side of (47), we get

$$
\begin{aligned}
& \int_{a}^{\sigma(s)} \int_{a}^{\sigma(r)} \frac{S_{t, r, s, \zeta} \phi(\Lambda(t)) \psi(\Omega(\zeta))}{(\sigma(t)-a)^{\frac{1}{2}}(\sigma(\zeta)-a)^{\frac{1}{2}}} \Delta t \Delta \zeta \\
\geq & \left(\int_{a}^{\sigma(r)}\left(\frac{\phi(\Phi(t))}{\Phi(t)}\right)^{2} \Delta t\right)^{\frac{1}{2}}\left(\int_{a}^{\sigma(r)} \int_{a}^{\sigma(\chi)}\left[\alpha(\theta) \phi\left(\frac{\lambda(\theta)}{\alpha(\theta)}\right)\right]^{2} \Delta \theta \Delta \chi\right)^{\frac{1}{2}} \\
& \times\left(\int_{a}^{\sigma(s)}\left(\frac{\psi(\Psi(\zeta))}{\Psi(\zeta)}\right)^{2} \Delta \zeta\right)^{\frac{1}{2}}\left(\int_{a}^{\sigma(s)} \int_{a}^{\sigma(z)}\left[\beta(\theta) \psi\left(\frac{\omega(\theta)}{\beta(\theta)}\right)\right]^{2} \Delta \theta \Delta z\right)^{\frac{1}{2}} \\
= & 2 M(r, s)\left(\int_{a}^{\sigma(r)} \int_{a}^{\sigma(\chi)}\left[\alpha(\theta) \phi\left(\frac{\lambda(\theta)}{\alpha(\theta)}\right)\right]^{2} \Delta \theta \Delta \chi\right)^{\frac{1}{2}} \\
& \times\left(\int_{a}^{\sigma(s)} \int_{a}^{\sigma(z)}\left[\beta(\theta) \psi\left(\frac{\omega(\theta)}{\beta(\theta)}\right)\right]^{2} \Delta \theta \Delta z\right)^{\frac{1}{2}} .
\end{aligned}
$$

From (45) and (46), the inequality (48) becomes

$$
\begin{aligned}
& \int_{a}^{\sigma(s)} \int_{a}^{\sigma(r)} \frac{S_{t, r, s, \zeta} \phi(\Lambda(t)) \psi(\Omega(\zeta))}{(\sigma(t)-a)^{\frac{1}{2}}(\sigma(\zeta)-a)^{\frac{1}{2}}} \Delta t \Delta \zeta \\
\geq & 2 M(r, s)\left(\int_{a}^{\sigma(r)}\left[\alpha(\chi) \phi\left(\frac{\lambda(\chi)}{\alpha(\chi)}\right)\right]^{2}(\sigma(r)-\chi) \Delta \chi\right)^{\frac{1}{2}} \\
& \times\left(\int_{a}^{\sigma(s)}\left[\beta(z) \psi\left(\frac{\omega(z)}{\beta(z)}\right)\right]^{2}(\sigma(s)-z) \Delta z\right)^{\frac{1}{2}},
\end{aligned}
$$


which is (40).

Remark 2. As a special case of Theorem 3 , when $\mathbb{T}=\mathbb{N}$, we can get (10) proved by Zhao and Cheung [12].

By applying Theorem 3 with $\phi(\chi)=\chi$ and $\psi(z)=z$, we can obtain the following theorem.

Theorem 4. Let $a \in \mathbb{T}$ and $\lambda, \omega$ be nonnegative functions. Then, the inequality

$$
\begin{aligned}
& \int_{a}^{\sigma(s)} \int_{a}^{\sigma(r)} \frac{S_{t, r, s, \zeta} \Lambda(t) \Omega(\zeta)}{(\sigma(t)-a)^{\frac{1}{2}}(\sigma(\zeta)-a)^{\frac{1}{2}}} \Delta t \Delta \zeta \\
\geq & (\sigma(r)-a)^{\frac{1}{2}}(\sigma(s)-a)^{\frac{1}{2}}\left(\int_{a}^{\sigma(r)} \lambda^{2}(\chi)(\sigma(r)-\chi) \Delta \chi\right)^{\frac{1}{2}} \\
& \times\left(\int_{a}^{\sigma(s)} \omega^{2}(z)(\sigma(s)-z) \Delta z\right)^{\frac{1}{2}},
\end{aligned}
$$

holds for all $r, s \in[a, \infty]_{\mathbb{T}}$, with

$$
S_{t, r, s, \zeta}=S\left(\frac{\int_{a}^{\sigma(r)} \lambda^{2}(\chi)(\sigma(r)-\chi) \Delta \chi}{(\sigma(r)-a)\left(\int_{a}^{\sigma(t)} \lambda^{2}(\chi) \Delta \chi\right)}\right) S\left(\frac{\int_{a}^{\sigma(s)} \omega^{2}(z)(\sigma(s)-z) \Delta z}{(\sigma(s)-a)\left(\int_{a}^{\sigma(\zeta)} \omega^{2}(z) \Delta z\right)}\right),
$$

and

$$
\Lambda(t)=\int_{a}^{\sigma(t)} S\left(\frac{(\sigma(t)-a) \lambda^{2}(\chi)}{\int_{a}^{\sigma(t)} \lambda^{2}(\chi) \Delta \chi}\right) \lambda(\chi) \Delta \chi, \Omega(\zeta)=\int_{a}^{\sigma(\zeta)} S\left(\frac{(\sigma(\zeta)-a) \omega^{2}(z)}{\int_{a}^{\sigma(\zeta)} \omega^{2}(z) \Delta z}\right) \omega(z) \Delta z
$$

Remark 3. As a special case of Theorem 4, when $\mathbb{T}=\mathbb{N}$, we can get (11) proved by Zhao and Cheung [12].

\section{Conclusions}

In this article, we first proved the reverse Hilbert-type inequalities on time scales which involving nonnegative and decreasing functions. After that, we proved the reverse Hilbert-type inequalities on time scales which involve nonnegative, concave, and supermultiplicative functions. All of these results are proved by using the Specht's ratio function. In future work, we will continue to generalize more the reverse Hilbert-type inequalities by using Kantorovich's ratio.

Author Contributions: Conceptualization, A.I.S., M.Z. and H.M.R.; Data curation, O.B., M.Z. and H.M.R.; Formal analysis, M.Z. and H.M.R.; Funding acquisition, G.A.; Investigation, G.A., H.A.A.E.-H. and O.B.; Methodology, G.A. and H.A.A.E.-H.; Visualization, H.M.R.; Writing-original draft, A.I.S., M.Z. and H.M.R.; Writing-review and editing, M.Z., H.M.R. and A.I.S. All authors read and agreed to the published version of the manuscript.

Funding: This research project was funded by the Deanship of Scientific Research, Princess Nourah bint Abdulrahman University.

Institutional Review Board Statement: Not applicable.

Informed Consent Statement: Not applicable.

Data Availability Statement: Not applicable.

Acknowledgments: This research project was funded by the Deanship of Scientific Research, Princess Nourah bint Abdulrahman University, through the Program of Research Project Funding After Publication, Grant No (PRFA-P-42-14).

Conflicts of Interest: The authors declare no conflict of interest. 


\section{References}

1. Hardy, G.H. Note on a Theorem of Hilbert Concerning Series of Positive Term. Proc. Lond. Math. Soci. 1925, 23, 45-46.

2. Hardy, G.H.; Littlewood, J.E.; Pólya, G. Inequalities, 2nd ed.; Cambridge University Press: Cambridge, UK, 1934.

3. Al-Smadi, M.; Arqub, O.A. Computational Algorithm for Solving Fredholm Time-Fractional Partial Integro-differential Equations of Dirichlet Functions Type with Error Estimates. Appl. Math. Comput. 2019, 342 , 280-294.

4. Al-Smadi, M.; Dutta, H.; Hasan, S.; Momani, S. On Numerical Approximation of Atangana-Baleanu-Caputo Fractional Integrodifferential Equations under Nncertainty in Hilbert Space. Math. Model. Nat. Phenom. 2021, 16, 41.

5. Hasan, S.; El-Ajou, A.; Hadid, S.; Al-Smadi, M.; Momani, S. Atangana-Baleanu Fractional Framework of Reproducing Kernel Technique in Solving Fractional Population Dynamics System. Chaos Solitons Fractals 2020, 133, 109624. [CrossRef]

6. Hasan, S.; Al-Smadi, M.; El-Ajou, A.; Momani, S.; Hadid, S.; Al-Zhour, Z. Numerical Approach in the Hilbert Space to Solve A fuzzy Atangana-Baleanu Fractional Hybrid System. Chaos Solitons Fractals 2021, 143, 110506. [CrossRef]

7. Hölder, O. Uber einen Mittelwerthssatz, Nachr. Ges. Wiss. Gott. 1889, 38-47.

8. Furuichi, S.; Minculete, N. Alternative Reverse Inequalities for Young's Inequality. arXiv 2011, arXiv:1103.1937.

9. Furuichi, S. Refined Young Inequalities with Specht's Ratio. J. Egyp. Math. Soc. 2012, 20, 46-49. [CrossRef]

10. Tominaga, M.; Specht's Ratio in the Young Inequality. Sci. Math. Japo. 2002, 55, 583-588.

11. Zhao, C.J.; Cheung, W.S. Hölder's Reverse Inequality and its Applications. Publ. l'Inst. Math. 2016, 113, 211-216. [CrossRef]

12. Zhao, C.J.; Cheung, W.S. Reverse Hilbert Type Inequalities. J. Math. Inequal. 2019, 13, 855-866. [CrossRef]

13. Ahmed, A.M.; AlNemer, G.; Zakarya, M.; Rezk, H.M. Some Dynamic Inequalities of Hilbert's Type. J. Func. Spac. 2020, $2020,4976050$. [CrossRef]

14. Rezk, H.M.; AlNemer, G.; Abd El-Hamid, H.A.; Abdel-Aty, A.H.; Nisar, K.S.; Zakarya, M. Hilbert-Type Inequalities for Time Scale Nabla Calculus. Adv. Differ. Equ. 2020, 2020, 619. [CrossRef]

15. El-Deeb, A.A.; Elsennary, H.A.; Cheung, W.-S. Some Reverse Hölder Inequalities with Specht's Ratio on Time Scales. J. Nonlinear Sci. Appl. 2018, 11, 444-455. [CrossRef]

16. Saker, S.H.; Ahmed, A.M.; Rezk, H.M.; O’Regan, D.; Agarwal, R.P. New Hilbert's Dynamic Inequalities on Time Scales. J. Int. Appl. 2017, 40, 1017-1039.

17. AlNemer, G.; Zakarya, M.; Abd El-Hamid, H.A.; Agarwal, P.; Rezk, H.M. Some Dynamic Hilbert-Type Inequality on Time Scales. Symmetry 2020, 13, 1410. [CrossRef]

18. Bohner, M.; Peterson, A. Dynamic Equations on Time Scales: An Introduction with Applications; Birkhäuser: Boston, MA, USA, 2001.

19. Bohner, M.; Peterson, A. Advances in Dynamic Equations on Time Scales; Birkhäuser: Boston, MA, USA, 2003.

20. Sandor, J. Inequalities for Multiplicative Arithmetic Functions. arXiv 2011, arXiv:1105.0292. 